\title{
Guide pour les pilules de contraception d'urgence : Introduire et développer l'offre des pilules de contraception d'urgence dans les pays en voie de développement
}

\author{
Sharif M.I. Hossain \\ Population Council \\ M.E. Khan \\ Population Council \\ Ricardo Vernon \\ Jill Keesbury \\ Population Council \\ lan Askew \\ Population Council
}

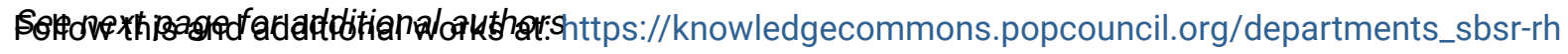

Part of the Demography, Population, and Ecology Commons, Family, Life Course, and Society Commons, Gender and Sexuality Commons, International Public Health Commons, Medicine and Health Commons, and the Women's Health Commons

How does access to this work benefit you? Let us know!

\section{Recommended Citation}

Hossain, Sharif M.I., M.E. Khan, Ricardo Vernon, Jill Keesbury, lan Askew, John Townsend, and Victoria Rumbold. 2009. "Guide pour les pilules de contraception d'urgence : Introduire et développer l'offre des pilules de contraception d'urgence dans les pays en voie de développement." Washington, DC: Population Council. 


\section{Authors}

Sharif M.I. Hossain, M.E. Khan, Ricardo Vernon, Jill Keesbury, Ian Askew, John Townsend, and Victoria Rumbold 


\section{Guide pour les Pilules de Contraception d'Urgence}

Introduire et développer l'offre des pilules de contraception d'urgence dans les pays en voie de développement 



\title{
Guide pour les Pillules de Contraception d'Urgence
}

Introduire et développer l'offre des pilules de contraception d'urgence dans les pays en voie de développement

\author{
Sharif M. I. Hossain \\ M.E. Khan \\ Ricardo Vernon \\ Jill Keesbury \\ Ian Askew \\ John Townsend \\ Victoria Rumbold
}


Les informations contenues dans ce guide reflètent les contributions de plusieurs personnes, en sus des auteurs. La plupart des informations sur les programmes de CU en Afrique, en Amérique latine et aux Caraïbes, proviennent de l'examen des publications réalisé respectivement par Jacinta Muteshi et Araceli Fernandez. La version finale du guide a été révisée par Patricia Stephenson (USAID), Nicole Gray (William and Flora Hewlett Foundation), Elizabeth Westley (Consortium International pour la Contraception d'Urgence) et Francine Coeytaux (Compton Foundation). Le guide a été traduit en français par Anne Verinaud. Il a été revu et corrigé par Nafissatou Diop et Youmane Faye (Population Council, Dakar).

Les auteurs et Population Council voudraient remercier l'USAID et la fondation William et Flora Hewlett pour leur soutien financier à la production de ce guide ainsi que leur engagement à faire connaître les PCU en Afrique et en Asie, mais également les Fondations Concept et Compton pour leurs idées et leur soutien à ECafrique.

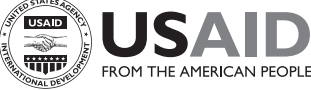

THE WILLIAM AND FLORA H E W L E T T F O U N D A T I O N
Ce guide a pu voir le jour grâce au généreux soutien du peuple américain via l'Agence des Etats-Unis pour le Développement International (USAID), l'Accord Coopératif Numéro HRN A-00-98-00012- et le Projet Numéro 2007-1124 de la fondation William et Flora Hewlett. Les contenus sont sous la responsabilité de Population Council et ne reflètent pas forcément les opinions de l'USAID, du Gouvernement des Etats-Unis ou de la Fondation William et Flora Hewlett.

Population Council mène des recherches dans le monde entier pour améliorer les politiques, les programmes et les produits dans trois domaines:le VIH et le SIDA, la pauvreté, le genre et les jeunes et la santé de la reproduction.

Le Programme Frontiers pour la Santé de la Reproduction (FRONTIERS) met en application des techniques de la recherche systématique pour améliorer la prestation de services de planification familiale et de santé de la reproduction, et influencer les politiques dans ce domaine. FRONTIERS est financé par l'Agence des Etats-Unis pour le développement international (USAID) et dirigé par Population Council en collaboration avec Family Health International.

Date de publication: Mars 2009

4301 Connecticut Avenue, Suite 280, Washington, DC

www.popcouncil.org

Proposition de Citation: Hossain, Sharif M.I. et al. 2009. «Guide pour les Pilules de Contraception d'Urgence (PCU): Introduire et développer l'offre des pilules de contraception d'urgence dans les pays en voie de développement, » Guide de FRONTIERS, Washington, DC: Population Council.

(c) 2009 The Population Council, Inc.

Population Council se réserve tous les droits de propriété de ce document. Aucune partie de cette publication ne peut être reproduite, conservée ou transmise sous quelque forme que ce soit, électronique, photocopie, enregistrement ou autre sans la permission de Population Council. 
Les pilules de contraception d'urgence (PCU) permettent à bien des femmes d'éviter une grossesse non désirée suite à l'échec d'une méthode contraceptive, d'un viol ou d'un rapport sexuel non protégé. L'accès à cette option sûre et efficace fait partie des éléments essentiels des soins de santé de la reproduction de qualité. Cependant, les PCU restent inaccessibles dans la plupart des pays du monde car les contraintes de l'offre et de la demande diminuent la possibilité pour les client(e)s d'utiliser la méthode de façon efficace. Ceci s'avère être le cas surtout dans les pays en voie de développement où les problèmes de stocks limités de produits, de compétence des prestataires, de la connaissance du public et de la mauvaise information se complètent régulièrement et limitent la disponibilité des PCU. Alors que les PCU ont été introduites de manière expérimentale dans beaucoup de pays, leur intégration effective dans les secteurs publics et privés a été retardée face à d'autres priorités concurrentes. Les interventions efficaces sont celles guidées par le principe que la fourniture des PCU n'est pas exclusivement la responsabilité des programmes de planification familiale du gouvernement. Ces interventions s'efforcent donc de généraliser la fourniture des PCU en développant les relations avec les fournisseurs et les pharmacies du secteur privé, le secteur $\mathrm{du} \mathrm{VIH} / \mathrm{SIDA}$ et les services pour les agressions sexuelles.

Comme pour l'introduction de la plupart des méthodes contraceptives dans un programme national, l'intégration efficace des PCU dans le système des soins de santé d'un pays est un projet complexe et en général de longue haleine. Ce guide a été conçu pour donner des conseils détaillés afin d'introduire et généraliser l'utilisation des PCU dans des pays aux contextes variés, de façon à répondre à la diversité de leurs besoins et de la disponibilité de leurs ressources.

La réalisation de ce guide a été le fruit de la collaboration de deux projets concernant des programmes pour la santé de la reproduction de Population Council: le projet de recherche opérationnelle en santé de la reproduction Frontiers (FRONTIERS), financé par l'USAID et le réseau d'ECafrique, ainsi que par la Fondation William et Flora Hewlett. Il synthétise les pratiques des programmes systématiquement documentés, tirées d'un ensemble d'expériences en Afrique, Asie, Amérique Latine et Caraïbes de ces dix dernières années. Le guide résume quatre études non publiées menées par FRONTIERS (Muteshi et Keesbury 2007; Fernández et Vernon 2007; Khan et Hossain 2007; Fernández et al. 2008) ainsi que des documents sélectionnés présentant des conseils supplémentaires propres à la fourniture des PCU après leur introduction et leur intégration (par exemple PATH 2004 et Pathfinder 2007).

Avec ce guide, nous espérons atteindre les responsables des programmes de santé de la reproduction (SR), les dirigeants gouvernementaux et les ONG. Il peut être utilisé dans les pays où les PCU ne sont pas disponibles pour le moment, dans des zones où le développement et la généralisation de l'accès aux services de PCU est en projet. Le guide:

$\checkmark$ prend en considération les besoins variés des différents groupes de la population et insiste sur certains «groupes spéciaux» comme les victimes de viol et les adolescents.

$\checkmark$ vise à présenter un processus global qui peut être adapté à des contextes spécifiques et qui se fonde sur des expériences efficaces et bien documentées dans des contextes divers.

$\checkmark$ oriente les dirigeants et responsables de programmes grâce au continuum des programmes pour les PCU: de l'évaluation des besoins et de la recherche opérationnelle, à l'enregistrement, la formation, la logistique et enfin la généralisation grâce à un développement à grande échelle au niveau national.

Le guide comporte quatre parties:

L'introduction et le contexte décrivent la situation actuelle des programmes pour les PCU dans le monde.

La réponse aux besoins de populations spécifiques décrit les démarches des programmes conçus pour des populations spécifiques.

L'intégration et la vulgarisation des PCU décrivent les cinq étapes chronologiques pour faire des programmes de PCU une composante intégrale d'un programme national de SR.

La Bibliographie qui propose une liste de ressources utiles pour les programmes de PCU. 
Introduction et contexte ................................................................................................ 1

Qu'est-ce que la contraception d'urgence? ..................................................... 1

Pourquoi les PCU sont-elles importantes? ...................................................... 1

Quand est ce qu'une femme a besoin des PCU? ........................................ 1

Les différentes PCU ........................................................................................... 1

L'efficacité et les effets secondaires des PCU .................................................. 2

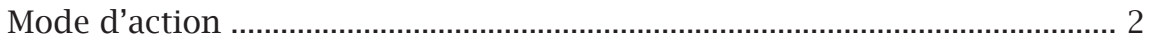

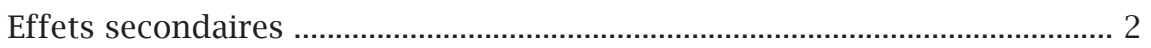

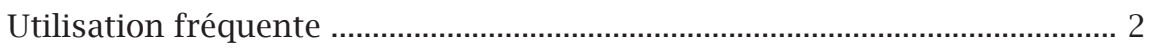

Les PCU et les IST, y compris le VIH ........................................................... 3

Que faire après avoir pris des PCU ............................................................. 3

Le rôle de «transition» des PCU ........................................................................ 3

L'offre de PCU dans des contextes variés ......................................................... 3

Répondre aux besoins de populations spécifiques .......................................... 5

Identifier les populations ayant des besoins spécifiques .............................. 5

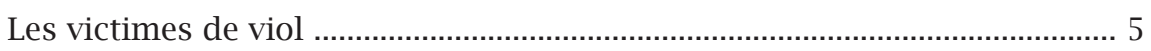

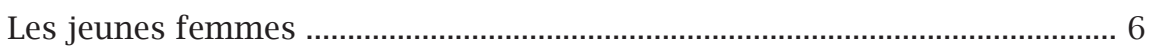

Les femmes vivant dans un contexte de crise humanitaire ........................ 8

Introduire et généraliser les PCU ................................................................................... 11

Etape 1. Enregistrement d'un produit de PCU spécialisé ............................... 11

Etape 2. Introduire les PCU dans le secteur public et auprès des ONG ....... 12

Etape 3. Former et renforcer les capacités des fournisseurs de PCU ............ 16

Etape 4. Défendre les PCU et sensibiliser le public ........................................ 19

Etape 5. Généralisation: Développement à grande échelle et institutionnalisation durable des services de PCU .......................................... 22

Bibliographie ............................................................................................................. 27 
ART

ARV

CAP

CDV

$\mathrm{CO}$

$\mathrm{COC}$

$\mathrm{CU}$

DIU

ICEC

IDP

IST

MS

MJTC

OMS

ONG

PCU

$\mathrm{PF}$

PPAG

PPC

PPE

SIDA

SIG

SR

UNFPA

$\mathrm{VIH}$
Traitement Antirétroviral

Médicaments antirétroviraux

Connaissances, Attitudes et Pratiques

Conseil et Dépistage Volontaire

Contraceptifs Oraux

Contraceptifs Oraux Combinés

Contraception d'Urgence

Dispositif Intra Utérin

International Consortium for Emergency Contraception

Personne déplacée à l'intérieur de son propre pays

Infection Sexuellement Transmissible

Ministère de la Santé

Mise à Jour sur la Technologie Contraceptive

Organisation Mondiale de la Santé

Organisation Non-Gouvernementale

Pilule Contraceptive d'Urgence

Planification Familiale

Planned Parenthood Ghana

Premier Point de Contact

Prophylaxie Post Exposition

Syndrome d'immunodéficience acquise

Système d'Information et de Gestion

Santé de la Reproduction

Fonds des Nations Unies pour la Population

Virus d'Immunodéficience Humaine 



\section{Qu'est-ce que la contraception d'urgence?}

La contraception d'urgence (CU) désigne les méthodes contraceptives qu'une femme peut utiliser dans les premiers jours suivant un rapport sexuel non protégé pour prévenir une grossesse non désirée (OMS 1998). La CU ne devrait être ni conseillée ni utilisée en tant que méthode contraceptive régulière. Les pilules contraceptives d'urgence (PCU) contiennent une plus forte dose d'hormones que les pilules contraceptives orales. Les PCU empêchent efficacement la grossesse lorsqu'elles sont prises dans les 120 heures (cinq jours) suivant le rapport non protégé, elles sont toutefois d'autant plus efficaces que prises le plus tôt possible. La PCU est parfois appelée à tort «la pilule du lendemain.»

\section{Pourquoi les PCU sont-elles importantes?}

$\checkmark$ Les PCU sont le seul moyen contraceptif pour empêcher une grossesse après un rapport non protégé.

$\checkmark$ Les PCU peuvent réduire le nombre de grossesses non désirées qui pourraient dans le cas contraire entraîner un avortement à risque.

$\checkmark$ Les PCU sont un élément important des soins post-viol et sont essentielles pour la santé de la reproduction.

\section{Quand est ce qu'une femme a besoin des PCU?}

Les PCU sont utilisées après un rapport sexuel non protégé. Ceci peut comprendre les situations suivantes:

$\checkmark$ Lorsqu'aucune méthode contraceptive n'a été utilisée;

$\checkmark$ Lorsqu'un préservatif se déchire;

$\checkmark$ Lorsqu'une femme oublie de prendre sa pilule contraceptive orale pendant deux jours ou plus, ou retarde l'injection programmée d'un contraceptif de plus de deux semaines;

$\checkmark$ Lorsqu'une femme est violée/forcée à avoir des rapports sexuels et n'utilise pas de méthode contraceptive à ce momentlà.

\section{Les différentes PCU}

1. La pilule progestative spécialisée (PCU spécialement libellée et emballée): $1.5 \mathrm{mg}$ de levonorgestrel en dose unique administrée dans les 120 heures suivant un rapport non protégé, en une ou deux pilules. Si deux pilules sont administrées (de $0.75 \mathrm{mg}$ chacune), elles peuvent l'être en même temps, donc en prise unique, ou bien les deux pilules peuvent être prises séparément mais à douze heures d'intervalle.

2. La méthode avec les contraceptifs oraux combinés (aussi connue sous le nom de méthode Yuzpe): au moins $100 \mathrm{mcg}$ d'ethinyl-œstradiol et 500mcg de levonorgestrel doit être prise dans les 120 heures suivant le rapport non protégé. La première dose est suivie de la seconde 12 heures plus tard. Cette méthode peut être utilisée en choisissant des pilules contraceptives adaptées et en calculant le nombre de pilules nécessaires à chaque prise.

3. Les contraceptifs oraux progestatifs: une prise de 20 à 25 pilules selon le dosage du type de pilules, à prendre dans les 120 heures suivant le rapport non protégé, la seconde prise de 20 à 25 pilules se fera 12 heures après. Cette option de PCU n'est en général pas recommandée étant donné le nombre important de pilules nécessaires par prise, mais elle peutêtre une option de secours en l'absence d'autres solutions.

D'après une étude multi centrique randomisée de l'OMS dans dix pays développés et en voie de développement, la dose unique de levonorgestrel de $1.5 \mathrm{mg}$ est aussi efficace que deux doses de $0.75 \mathrm{mg}$ prises à 12 heures d'intervalle pour réduire le risque de grossesse. Les effets secondaires étaient les mêmes pour les deux méthodes (von Hertzen et al. 2002). Selon le Consortium International pour la Contraception d'Urgence (ICEC), «les prestataires devraient conseiller aux femmes de prendre une dose unique de $1.5 \mathrm{mg}$ de levonorgestrel» (ICEC 2003a). L'option \# 1 simplifie l'utilisation de la PCU mais si elle n'est pas disponible, les options \#2 et \#3 devraient être envisagées. 


\section{L'efficacité et les effets secondaires des PCU}

L'efficacité des PCU pour prévenir une grossesse est moindre que celle des méthodes de contraception régulières. Les chiffres ci-dessous reflètent le risque de grossesse dans les 120 heures qui suivent un rapport non protégé.

\section{Le progestatif seul:}

$\checkmark$ Réduit le risque de grossesse d'un seul rapport non protégé de 60 à 90 pour cent (OMS et al 2005).

$\checkmark$ Effets secondaires: Nausées pour 23 pour cent des femmes et vomissements pour 6 pour cent (OMS 1998).

\section{Le combiné progestatif et œstrogène:}

$\checkmark$ Réduit le risque de grossesse d'un seul rapport non protégé de 75 pour cent.

$\checkmark$ Effets secondaires: Nausée pour 43 pour cent des femmes et vomissements pour 16 pour cent (ICEC 2004).

\section{Mode d'action}

Le mode d'action précis des PCU dépend de la date du rapport sexuel dans la phase du cycle menstruel de la femme et de celle de la prise des PCU. Les PCU ne peuvent pas interrompre une grossesse en cours ni être néfastes pour le développement de l'embryon. Selon une déclaration conjointe d' ICEC et de la Fédération Internationale de Gynécologie et d'Obstétrique (FIGO), les pilules contraceptives contenant uniquement le levonorgestrel empêchent la grossesse en:1) inhibant ou retardant l'ovulation si elles sont administrées avant l'ovulation; ou 2) en empêchant la fécondation en influant sur le mucus cervical ou sur la capacité du sperme à se fixer à l'ovule.

"La contraception d'urgence n'est pas un avortement médical précoce. La CU n'est efficace que dans les premiers jours suivant le rapport, avant que l'ovaire ne libère l'ovule et avant que le sperme ne féconde l'ovule. L'avortement médical est un choix pour les femmes au début d'une grossesse en cours mais cela nécessite un médicament différent du levonorgestrel» (ICEC et FIGO 2008).

La plupart des études ont montré que les PCU contenant uniquement le levonorgestrel ne changeaient pas les caractéristiques histologiques ou biochimiques de l'endomètre, ce qui indique qu'elles ne peuvent pas empêcher l'implantation. (ICEC et FIGO 2008).

Il est essentiel de savoir comment agissent les PCU pour comprendre la différence essentielle entre la contraception d'urgence et un avortement médical précoce. La confusion entre les deux a souvent entraîné un rejet ou une dénonciation injustifiée des PCU. Tout d'abord, il s'agit de dosages et de catégories de médicaments différents. Ensuite, les PCU ne sont efficaces que dans les 120 heures suivant le rapport sexuel, avant l'implantation de l'ovule fécondé dans l'utérus, ce qui détermine le début de la grossesse selon la définition médicale. A l'opposé, l'avortement médical est l'interruption précoce d'une grossesse par l'expulsion de l'ovule implanté et fécondé.

Les PCU ne devraient pas être confondues avec le misoprostol, le mifépristone ou le RU486 qui ont effectivement un effet abortif.

\section{Effets secondaires}

Les effets secondaires principaux signalés comprennent:

$\checkmark$ Les nausées et les vomissements qui sont les effets secondaires les plus courants.

$\checkmark$ Les saignements intermittents.

$\checkmark$ D'autres effets sont possibles comme les céphalées, les vertiges, la fatigue et la tension des seins.

La plupart des effets secondaires disparaissent dans les 24 heures suivant la prise du médicament. Pour réduire les nausées, les PCU peuvent être prises pendant les repas et les antiémétiques peuvent aider les femmes qui ont généralement des nausées ou des vomissements quand elles prennent des pilules contenant des hormones. Si une femme vomit moins de deux heures après la prise de la PCU, elle devrait reprendre une pilule. En cas de vomissements importants, la CU peut être administrée par voie vaginale (ICEC 2004).

\section{Utilisation fréquente}

Il n'existe aucune preuve que l'utilisation fréquente des PCU soit néfaste pour la plupart des femmes (Abuabara et al. 2004), excepté un risque accru d'une grossesse non désirée si les PCU sont utilisées habituellement à la place d'une méthode contraceptive régulière. Les PCU sont sans risque même si elles sont utilisées plus d'une fois dans le cycle (ICEC 2003b); cependant l'utilisation répétée des 
PCU n'est pas recommandée pour les femmes qui ont des rapports sexuels fréquents ou qui peuvent être exposées au risque d'infection par les IST/VIH. D'autres méthodes contraceptives sont plus efficaces pour éviter une grossesse et aussi plus abordables, les préservatifs ont également l'avantage de protéger contre les IST. Si une cliente signale qu'elle utilise les PCU fréquemment, on recommande au prestataire de santé de lui conseiller d'adopter une méthode contraceptive régulière.

\section{Les PCU et les IST, y compris le VIH}

Les PCU ne protègent pas contre les IST, y compris le VIH. Si une femme a été violée, il est important de lui donner une prophylaxie post exposition (PPE) pour les IST, dans les 72 heures suivant l'incident. La prophylaxie habituelle pour le VIH est un sujet de controverse et ne constitue pas une norme de pratique acceptée à l'unanimité. Le personnel de santé devrait se référer aux protocoles locaux sur la PPE pour le VIH s'ils existent (OMS 2003a).

\section{Que faire après avoir pris des PCU}

La fourniture des PCU peut être l'occasion pour les prestataires de conseiller les client(e)s sur des comportements sexuels ultérieurs sans risque. Ils devraient recommander aux client(e)s :

$\checkmark$ D'utiliser une méthode contraceptive de protection ou de s'abstenir de rapport sexuel jusqu'aux prochaines règles.

$\checkmark$ De ne pas utiliser les PCU comme une méthode contraceptive régulière.

$\checkmark$ D'adopter une méthode contraceptive régulière (voir le tableau ci-dessous).

Les prestataires de services peuvent également conseiller ou orienter les client(e)s vers la planification familiale ou d'autres services de santé de la reproduction, en particulier pour les victimes de viol qui ont besoin d'une prise en charge médicale et psycho-sociale immédiate (voir ci-dessous).

\begin{tabular}{|l|l|}
\hline Méthode & Quand commencer \\
\hline Préservatif & $\begin{array}{l}\text { Immédiatement après l'utilisation } \\
\text { des PCU - les préservatifs protègent } \\
\text { doublement contre la grossesse et le } \\
\text { VIH/IST }\end{array}$ \\
\hline Orale & $\begin{array}{l}\text { Le jour suivant la 2ème prise de PCU } \\
\text { ou de } 1 \text { à } 7 \text { jours après les règles }\end{array}$ \\
\hline Injectable & \\
\hline DIU & De 1 à 7 jours après les règles \\
\hline Implant &
\end{tabular}

\section{Le rôle de «transition» des PCU}

Faire la transition vers des méthodes contraceptives régulières

La fourniture des PCU donne l'occasion d'inciter les client(e)s à utiliser une méthode contraceptive régulière et plus efficace en communicant des informations sur d'autres méthodes disponibles, sur l'importance de la protection mutuelle (comprenant les préservatifs masculins et féminins) ainsi que sur l'utilisation correcte et régulière de la contraception. Une étude au Bangladesh a montré que suite à une première utilisation des PCU (surtout due à l'échec de la méthode utilisée), la proportion des femmes qui utilisaient une méthode régulière de contraception avait augmenté de 72 à 93 pour cent (Khan et Hossain 2008). En Inde, le suivi des utilisateurs six mois après leur première utilisation des PCU a relevé une hausse significative de 67 à 79 pour cent concernant l'utilisation d'une méthode de planification familiale régulière (Kumar et al. 2007).

\section{Faire la transition vers d'autres services de santé de la reproduction}

L'offre et l'utilisation des PCU constituent une occasion pour communiquer un nombre de messages et de services sur la santé sexuelle et de la reproduction aux client(e)s et également les orienter vers d'autres services de santé de la reproduction. Au Kenya par exemple, 62 pour cent des prestataires de PF offraient des services complémentaires à leurs client(e)s de CU sans que leur formation ait insisté sur la «transition». La plupart du temps, ceux-ci incluaient des conseils sur le VIH/IST ou une orientation vers un centre de dépistage. On peut noter que presque la moitié des personnes conseillées sur la PF ont accepté une méthode nouvelle ou différente, en général des contraceptifs injectables ou des préservatifs masculins (Keesbury, Owino et Bradford 2007). L'offre des PCU peut également renforcer les efforts pour la prévention du VIH. Les PCU peuvent de même servir comme méthode de secours indispensable pour les femmes séropositives, les couples en désaccord en cas d'échec de la méthode, ainsi que ceux qui souhaitent éviter une éventuelle transmission de la mère à l'enfant (Townsend et al. 2007).

\section{L'offre de PCU dans des contextes variés}

La contraception d'urgence est à présent disponible pour les femmes dans plus de 140 pays dans le monde et en vente libre dans les pharmacies dans 44 pays (ICEC 2008). En fonction des lois nationales, elle peut être fournie par le gouvernement, les ONG et les cliniques 
privées, les pharmacies et docteurs du secteur privé, les agents de santé communautaires et d'autres lieux de prestations. Les points d'accès varient selon l'importance des systèmes de santé publique et la pénétration du marché privé. En Asie du sud par exemple, la CU est fournie essentiellement grâce aux programmes gouvernementaux de planification familiale en place. En Afrique, les pharmacies sont les sources de fournitures habituelles et en Amérique latine et aux Caraïbes, elle peut être trouvée dans des points de vente à la fois publics et privés.

Cependant, peu de pays en voie de développement peuvent se prévaloir d'avoir fait des PCU un service intégral et disponible rapidement au sein du programme national de SR. Les PCU existent en Argentine, au Brésil, au Mexique, au Pérou et au Bangladesh. Dans plusieurs pays, la méthode est devenue plus accessible, surtout à travers les secteurs commerciaux et les ONG plutôt que dans le secteur public. Toutefois, pour la majorité des pays, la PCU est disponible notamment pour répondre aux besoins des femmes dans certaines situations, tel que les cas de viol ou dans le contexte universitaire. Cette diversité incite à 'associer les PCU seulement à des groupes de femmes définis ayant des besoins spécifiques (en Equateur, le secteur public fournit des PCU seulement aux victimes de viol) et non comme une méthode qui pourrait être utilisée par la population dans son ensemble suite à un rapport non protégé ou à l'échec de la méthode. Dans les pays où l'opposition politique et religieuse est fermement contre une plus grande disponibilité des PCU son introduction en tant que service pour des femmes particulièrement vulnérables, comme les victimes de viol, peut servir de base pour développer par la suite l'offre à plus grande échelle.

Le but principal de ce guide est de fournir des conseils sur les démarches des programmes visant à généraliser les PCU au sein d'un programme national de SR afin qu'elles deviennent aussi accessibles que les autres méthodes contraceptives. Les PCU sont souvent introduites dans un pays pour un groupe spécifique de femmes ayant des besoins non satisfaits plus importants que le reste de la population. La partie qui suit analyse les enseignements tirés des programmes de services pour les PCU concernant les femmes ayant des besoins spécifiques. Il s'agit des jeunes femmes non mariées, des victimes d'agression sexuelle et des femmes déplacées vivant dans des camps de réfugiés.

Après l'étude globale des programmes pour les femmes dans des situations spécifiques, la plus grande partie de ce guide sera consacrée aux étapes stratégiques qui sont essentielles lorsqu'on s'efforce de généraliser les PCU en vue d'accroître leur disponibilité pour le grand public. Il est clair que la plupart des pays ont besoin de généraliser l'offre des PCU pour accroître l'accès au grand public tout en mettant simultanément en œuvre des programmes qui peuvent répondre aux besoins des femmes dans des situations spécifiques. Ce guide vise à fournir des conseils qui peuvent être utiles aux dirigeants et responsables de programmes de SR pour atteindre ces deux objectifs. 


\section{Répondre aux besoins de populations spécifiques}

\section{Identifier les populations ayant des besoins spécifiques}

Une grossesse non désirée peut représenter un fardeau pour n'importe quelle femme, quelque soit sa situation. Cependant, certaines femmes dans des situations particulières sont plus vulnérables que d'autres, à la fois individuellement et socialement. Pour elles, la CU peut jouer un rôle particulièrement important en préservant leur bonne santé physique et psychologique tout en leur évitant une grossesse non désirée. Dans cette partie nous étudions trois groupes de populations dont les besoins seraient mieux satisfaits s'ils recevaient des PCU à travers des systèmes d'offre spécifiques: les victimes de viol, les jeunes femmes non mariées et les femmes vivant dans des camps de réfugiés.

\section{Les victimes de viol}

Une grossesse suite à un viol est en général toujours non désirée et traumatisante. De manière générale, les PCU devraient être disponibles pour les femmes victimes de viol qui sont en âge de procréer ou qui montrent des signes de caractères sexuels secondaires, ou des femmes qui ne sont pas enceintes et qui n'utilisent pas une méthode efficace de contraception (OMS 2003a). Un test de grossesse n'est pas nécessaire avant de fournir la CU après un viol, mais la grossesse peut être écartée en utilisant une liste de contrôle simple (FHI 2007). Dans tous les cas, le prestataire devrait affirmer au client que même si la femme est enceinte, l'utilisation des PCU ne sera pas néfaste pour le foetus. Comme la CU doit être administrée dans les 120 heures suivant le viol et qu'elle est d'autant plus efficace si elle l'est le plus tôt possible, tous les messages d'information sur les services post-viol devraient insister sur l'importance cruciale d'obtenir les PCU (et dans les contextes de prévalence du VIH élevée, une PPE-VIH également) aussi vite que possible après le viol (Keesbury, Skibiak et Zama 2006; Keesbury et Zama 2007; Vernon, Schiavon, et Llaguno 1997).

Le créneau restreint pendant lequel on peut espérer réduire le risque d'une grossesse après un viol fait que les services et l'information pour les PCU devraient être fournis aux victimes de viol dès leur premier point de contact (PPC) ou dès que possible par la suite. Le premier point de contact peut varier considérablement, mais c'est en général: (1) L'endroit où le crime est rapporté en premier, le plus souvent au poste de police, dans les bureaux des services sociaux ou du ministère public, des agences d'aide juridique ou les refuges pour les femmes; (2) l'endroit où la victime se rend pour être soignée (soit avant ou après avoir déclaré le crime), telles que les unités d'urgence ou de consultation externe des établissements de santé. Certains pays ont, ou sont en train de développer des centres spécialisés qui peuvent apporter toute l'aide médicale, juridique, psychologique et autre pour les victimes dans un seul point de contact (par ex. le Thuthuzela Rape Centers en Afrique du Sud [UNICEF 2008]).

Dans plusieurs pays (ex. L'Equateur, le Guatemala, le Mexique et la Zambie), les conseils et services pour les PCU ont été introduits dans les circonscriptions de la police et dans les centres médicaux pour les victimes de viol. L'expérience montre que le personnel de ces centres a besoin d'une formation plus approfondie que le personnel de santé dans le domaine du conseil aux victimes de viol et peut avoir besoin du soutien et de l'accord spécifique du secteur de la santé. Il est essentiel d'inclure ce service dans les directives et les protocoles pour s'assurer que le personnel fournisse ces services systématiquement. Lorsque les PCU ne sont pas fournies directement, ces PPC devraient orienter les victimes vers les pharmacies ou les cliniques où elles peuvent obtenir les pilules et des instructions écrites sur leur utilisation, si elles existent, ou encore vers les centres médicaux pour les victimes de viol où un plus grand nombre de besoins peuvent être traités (Vernon, Schiavon, et Llaguno 1997; Taracena et Brambila 2001 ; Keesbury et Zama 2007).

Mettre en place des services de PCU pour les victimes de viol dans ces PPC peut nécessiter la formation de prestataires comme les agents communautaires ou les prestataires de santé sur les lieux de travail selon les directives nationales. Les employés et les responsables devraient au minimum suivre les formations portant sur les points suivants:

$\checkmark$ Les caractéristiques des PCU

$\checkmark$ Identifier quelles victimes de viol devraient recevoir les PCU

$\checkmark$ Conseiller les victimes sur leurs options et leur fournir des PCU ou les orienter 


\section{Fournir les PCU dans le premier point de contact pour les victimes de viol}

En Zambie, la majorité (91\%) des victimes de viol de la province du Copperbelt se tournent vers la police avant de solliciter des soins (Keesbury et Zama 2007). De plus, bien que 82 pour cent de toutes les victimes aient réussi à se rendre dans un centre de soins dans le temps imparti pour les PCU, seules 37 pour cent ont en réalité reçu des PCU du personnel de l'hôpital. Ces résultats ont encouragé le Ministère de la Santé à former des officiers de police comme distributeurs communautaires pour les PCU. Des estimations suggèrent d'autoriser les policiers à fournir des PCU, ceci ferait bénéficier jusqu'à 59 pour cent des victimes de viol, avec une petite différence entre les zones rurales ou urbaines (Keesbury, Skibiak, et Zama 2006).

La fourniture des PCU dans les PPC par un personnel non médicalement qualifié pose toutefois certains problèmes. Au Bangladesh et en Inde, bien que les postes de police soient des PPC assez fréquentés, la police refuse de stocker ou de fournir des PCU; elle accepte seulement avec réticence de donner des informations sur les PCU aux victimes (Khan et al. 2008).

Fournir des brochures sur les PCU dans les PPC plutôt que les pilules elles-mêmes était une pratique courante au Mexique car en l'absence de directives officielles, le personnel des établissements publics craignait de se voir inculpé pour avoir pratiqué des avortements et fourni des médicaments sans diplôme médical (Vernon, Schiavon et Llaguno 1998). Même lorsque les PPC non-médicaux s'engagent à fournir des PCU, la conformité aux services de fourniture peut varier de manière significative, le suivi et la supervision des activités sont donc nécessaires.

Après la fourniture des PCU, les orienter vers une PPE -IST/VIH et un service pour traiter leur traumatisme au niveau médical et psychologique (si cela n'a pas été déjà fait)

$\checkmark$ En fonction des préférences individuelles et du cadre juridique national, l'ouverture ou l'orientation vers le service approprié pour l'ouverture d'un dossier pénal.

L'offre des PCU dans le PPC est fortement plus recommandée que l'orientation vers un autre établissement en raison de la nécessité d'utiliser les PCU immédiatement, surtout dans les cas où les transports ne sont pas tout de suite disponibles (Vernon, Schiavon, et Llaguno 1997; Vernon 1998; Kestler et Ramirez 2004; Keesbury et Zama 2007).

Les femmes victimes de violence sexuelle gardent souvent des blessures physiques et après l'agression, présentent plus de risques que les autres femmes d'avoir une grossesse non désirée, de se plaindre de symptômes d' infection de l'appareil reproducteur, d'avoir des partenaires multiples et d'être moins susceptibles d'utiliser des préservatifs et d'autres contraceptifs (IFPP 2004). La CU est seulement l'un des éléments d'un ensemble de services post-viol nécessaires pour réduire les diverses conséquences possibles de la violence sexuelle. Autant que possible, un ensemble de soins complets et intégrés devrait être disponible soit dans un lieu unique (ex. le modèle de Thuthuzela) ou, plus couramment, à travers un système d'orientations coordonnées au sein des unités spécialisées.

\section{Les jeunes femmes}

Le terme «jeunes» fait référence, selon la définition de l'OMS, aux individus âgés de 10 à 24 ans, cela inclut donc les adolescent(e)s (de 10 à 19 ans) et les jeunes adultes (de 15 à 24 ans). Pour la plupart des jeunes gens, c'est le temps de l'initiation sexuelle et des expériences, souvent caractérisées par des rapports peu fréquents, non prévus et parfois non consensuels. De par sa nature même, ce type d'activité sexuelle est souvent non protégé ou insuffisamment protégé contre la grossesse et les IST. Assurer l'accès et l'utilisation des PCU peut être un élément essentiel des efforts principaux de tout programme de SR pour réduire les grossesses non désirées au sein de cette population. Les jeunes gens constituent alors une importante et croissante population à atteindre par des services de PCU d'un programme national de SR. Des témoignages du Kenya, montrent que les jeunes constituent jusqu'à 80 pour cent de tous les clients de PCU du secteur public et 49 pour cent de ceux qui achètent les pilules en pharmacies (Keesbury, Owino, et Bradford 2007).

Les jeunes ne forment pas un groupe homogène, même au sein d'un seul pays. Pour les atteindre, les stratégies des programmes de PCU doivent donc être flexibles. Dans la plupart des cas, les informations et les services de PCU devraient être disponibles dans les mêmes structures et programmes que les autres services et informations de santé de la reproduction à destination des jeunes, telles que les cliniques adaptées aux adolescent(e)s, les programmes communautaires et parfois, les programmes scolaires. Etant donné que beaucoup d'adolescent(e)s peuvent être intimidés ou ne pas 
faire confiance aux services en place, ou n'ont pas beaucoup d'argent et ont moins de connaissances que les personnes plus âgées, il est d'autant plus important que les services de PCU pour les jeunes comprennent les caractéristiques recommandées pour les services de contraception de qualité en général, y compris:

\section{$\checkmark$ L'intimité et la confidentialité \\ $\checkmark$ Un accès facile \\ $\checkmark$ Des services et des produits à un prix raisonnable \\ $\checkmark$ Des horaires pratiques et flexibles \\ $\checkmark$ Un personnel sensible, d'un abord facile et ayant été formé à conseiller des adolescent(e)s}

Les programmes pour les PCU dans les structures déjà en place nécessitent une planification, des prévisions budgétaires, la mise en place des activités qui permettent d'inclure des messages et des informations dans les stratégies de communication, la formation des personnels sur place, la fourniture et la conservation faciles des stocks de produits. Par exemple, dans les écoles secondaires, les informations relatives aux PCU pourraient être incluses dans un curriculum plus large couvrant la santé sexuelle et reproductive ou lors de sessions pour les étudiants, professeurs et parents, consacrées spécialement aux PCU qui incluraient ce sujet. Dans les établissements scolaires du supérieur et certains du secondaire, les services de santé actuels pour les étudiants peuvent être aménagés pour fournir des PCU, parallèlement à des conseils et des services pour la contraception et la prévention du VIH/IST. Pour de plus amples informations sur les services adaptés aux jeunes, voir YouthNet/ FHI (2004) et pour des ressources permettant de guider les programmes sur les PCU à l'endroit des jeunes; voir Beitz et al. (2003) et Parker (2005).

Intégrer les services de PCU à des activités appropriées pour les jeunes peut également s'avérer être un bon moyen d'augmenter le nombre de messages sur les PCU et leur impact. Toutefois, un projet ou un programme se focalisant uniquement sur les PCU pour les adolescent(e)s et les jeunes n'est souvent ni accepté, ni faisable dans de nombreux pays.

Ci-dessous, des méthodes supplémentaires pour atteindre les jeunes avec des informations sur les PCU:

$\checkmark$ Identifier et utiliser les médias et les chaînes d'information auxquels les jeunes ont accès. Par exemple: les journaux, les services d'assistance téléphonique, les revues pour les jeunes,

\section{Atteindre les jeunes avec des informations sur les PCU}

Une étude en Zambie a montré que les adolescent(e)s, les pharmaciens et les jeunes pairs éducateurs représentaient la source d'informations la plus fréquente sur les PCU, plus que les prestataires des cliniques ou les agents communautaires distributeurs de contraceptifs (Skibiak, Chambeshi-Moyo, et Ahmed 2001).

Au Mexique, I'IPPF affilié à MEXFAM, a introduit les PCU dans les programmes de l'ensemble des cours de formation de santé de la reproduction pour les écoles et les communautés, et a initié les services pour les PCU dans les cliniques adaptées aux adolescent(e)s ainsi que dans les programmes communautaires et d'éducation par les pairs. La connaissance des PCU parmi les jeunes a considérablement augmenté (Vernon et Durá 2004). L'affichage de posters, la distribution de cartes postales et la création de lignes d'assistance téléphonique se sont avérés être des moyens efficaces pour informer les jeunes (Givaudan, Fuertes, et Vernon 1998; Vernon 1998; Ellertson et al. 2002).

En Inde, les PCU apparaissent maintenant régulièrement dans les publicités sur de nombreuses chaînes de télévision et sur les ondes des stations de radio populaires auprès des jeunes. Le Ministère de la Santé et du Bien-être Familial (MOHFW) met en place des cliniques pour la santé sexuelle et reproductive adaptées aux adolescent(e)s dans les établissements ruraux de santé et dans les hôpitaux urbains de district. Le conseil et l'offre des PCU en sont des composantes clefs (MOHFW 2006).

Au Kenya, l'expérience de la formation des pharmaciens en salle n'a pas été satisfaisante en raison du renouvellement fréquent des pharmaciens et des départs prématurés au cours de la formation. L'étude en a conclu que la formation et la distribution de matériels d'IEC sur site seraient plus efficaces (Beitz et al. 2003). Une autre étude au Kenya a révélé que les pharmaciens ne s'engagent pas dans des discussions plus approfondies avec leurs clients de CU sur leur santé et perdent donc ainsi l'occasion de discuter des IST et d'autres problèmes importants de SR (Keesbury et al.).

En Bolivie, la formation sur site du personnel des pharmacies pour fournir des informations appropriées et adaptées aux adolescent(e)s ainsi que des services grâce à des jeux de rôles avec des groupes d'adolescent(e)s a augmenté significativement les ventes de PCU (Arteaga et La Fuente 2001). 
les sites web et publicités pour des événements destinés aux jeunes.

$\checkmark$ Faire des PCU un produit en vente libre disponible pour les jeunes. Ceci réduirait considérablement les obstacles médicaux pour accéder aux PCU.

$\checkmark$ Encourager et former les pharmaciens à stocker les PCU et à diffuser des informations exactes à leurs client(e)s, aux jeunes en particulier.

\section{Les femmes vivant dans un contexte de crise humanitaire}

On sait relativement peu de choses sur la meilleure manière de fournir des services de santé de la reproduction, y compris les PCU, dans un contexte de crise humanitaire. La fourniture de services aux réfugiés et aux personnes déplacées à l'intérieur de leur propre pays (IDP) est en général adaptée aux modèles conçus pour des communautés stables et il existe peu d'informations sur la manière dont ces exemples d'offre de services peuvent être utilisés au mieux ou adaptés aux besoins des réfugiés ou IDP. De plus, le statut et les besoins en santé de la reproduction d'une femme réfugiée vivant à long terme dans un camp peuvent être très différents de ceux des réfugiés pendant la phase d'urgence de certaines crises, ou de ceux des IDP et d'autres groupes affectés par la guerre (McGinn 2000). Cette partie étudie certains des problèmes associés aux programmes pour les PCU dans ce type de contexte.

Les situations d'urgence humanitaires peuvent arriver brusquement, et de par leur nature, il est important de s'allouer du temps pour une formation intensive et complète des prestataires. Pourrait inclure le personnel médical et paramédical ainsi que les accoucheurs et accoucheuses traditionnels, le personnel formé sur le terrain et les bénévoles. Idéalement, les prestataires devraient être formés et sensibilisés à l'avance ou, si nécessaire, par un conseiller spécialisé en SR après que la réponse soit lancée (Goodyear et McGinn 1998). Le personnel médical devrait être formé à donner des conseils sur les PCU, à faire passer des tests pour les IST et aux soins prophylactiques pour les victimes de viol avant la prise en charge indispensable de leur traumatisme.

Des programmes-réponses peuvent être nécessaires pour se montrer créatif dans la recherche des conseillers. Dans les camps de Kibondo en Tanzanie, les responsables élues par les femmes réfugiées dans les camps ont été formées pour faire de l'éducation communautaire et pour conseiller les victimes de viol. Les

\section{Kits sanitaires pour les situations d'urgence: où s'approvisionner}

\author{
Le Reproductive Health Response in Conflict \\ Consortium. 2008. La contraception d'urgence \\ pour les zones touchées par des conflits. Module \\ d'apprentissage à distance. Pour commander \\ des exemplaires imprimés, envoyez un courrier \\ électronique à info@rhrc.org. Disponible à: http:// \\ rhrc.org/resources/general_fieldtools/er_contraception/ \\ ec_brochure_French.pdf \\ Site web: www.rhrc.org
}

Organisation Mondiale de la Santé. 1999. Nouveau kit sanitaire d'urgence 98: Médicaments et matériel médical pour une population de 10000 personnes pendant environ 3 mois. Disponible à: http://apps. who.int/medicinedocs/en/d/Jwhozip29f/

Site web: www.who.org

Organisation Mondiale de la Santé. 2005. Gestion clinique des Victimes de Viol Développement de protocoles à adopter avec les réfugiés et les personnes déplacées dans leur propre pays, Edition révisée. Disponible à: http://whqlibdoc.who.int/ publications/2005/9242592633_fre.pdf

Site web: www.who.org

accoucheurs et accoucheuses traditionnels et les para professionnels des services sociaux ont reçu une formation pour sensibiliser sur la violence, et fournir des informations de base sur les PCU (Goodyear et McGinn 1998).

Les PCU devraient être immédiatement disponibles au début de la réponse à une crise humanitaire. Le groupe de travail inter-agences de l'OMS sur la santé génésique dans les situations de réfugiés (OMS 2003b) a développé un ensemble de services initiaux minimum (Minimum Initial service Package -MISP) pour répondre aux besoins de santé de la reproduction des femmes et jeunes filles déplacées, qui inclut des PCU parmi les services à fournir. L'OMS a également inclus les PCU dans le «nouveau kit de santé d'urgence,» un ensemble de produits de base délivré immédiatement à chaque site d'urgence nouvellement identifié. Le personnel des programmes travaillant dans des contextes d'urgence devrait être conscient des besoins pour trouver, commander et distribuer les PCU assez tôt dans la période de crise. Ceci peut être négligé dans l'agitation des premiers jours.

Les principales barrières à l'offre des PCU dans un contexte de crise humanitaire sont les mêmes que pour la population en général: le manque de connaissance des femmes et des prestataires de santé sur les PCU, la stigmatisation des utilisateurs, des stocks inadaptés et l'indifférence 
du prestataire ou convictions. Comme dans la population en général, ces barrières entraînent une sous-utilisation de PCU. Les données des camps de réfugiés de sept pays montrent que 54 pour cent des femmes victimes de viol n'ont pas reçu les PCU dans les 120 heures suivant l'incident en 2007 (UNHCR 2008). En gardant à l'esprit qu'il est important de considérer que pour garantir l'observance, des PCU spécialisées en dose unique peuvent être plus adaptées pour des client(e)s ponctuelles.

La compréhension et les connaissances limitées sur la PCU dans les camps de réfugiés reflètent souvent celles des populations en situation de pré-crise. Une étude dans le camp de Kakuma au Kenya a montré que, comme dans la population en général, les PCU étaient largement perçues comme étant abortives. De plus, sur 34 pour cent des femmes et 27 pour cent des hommes qui étaient au courant de cas de viol, seuls 11 pour cent savaient que la contraception d'urgence était disponible dans les services de santé du camp, démontrant ainsi le besoin de communiquer plus d'informations aux populations réfugiées (Muia, Fikree, et Olenja 2000). Après la formation sur la CU des prestataires de santé, des dirigeants des groupes de soutien pour les femmes, des responsables de la santé communautaire, des agents de santé communautaires et des représentants de la planification familiale à Kakuma, la connaissance de la méthode a plus que doublé au sein de la population réfugiée, de 15 à 35 pour cent (Muia et al. 2002).

Une équipe œuvrant dans la réponse humanitaire devrait sans doute prendre en considération les lois et protocoles nationaux à l'avance et progresser pour promouvoir le changement, si cela est approprié. Par exemple, les ONG au Soudan expliquent qu'elles ne font pas de publicité pour les services destinés aux victimes de viol car la loi soudanaise (Article 48) interdit aux docteurs de soigner les victimes de viol si les patients n'ont pas été recommandés par le département de la police. Les docteurs et le personnel de santé peuvent être arrêtés pour violation de cette loi, et certains ont été menacés de devoir arrêter d'offrir leurs services. Dans le camp de Kakuma au Kenya, bien que les prestataires formés aient accru la connaissance sur la CU parmi les réfugiés, les services de CU n’ont pas pu être institutionnalisés et les services pour la contraception ont continué à être fournis comme avant la formation (Muia et al. 2002). 


\section{Introduine et généraliser les PCU}

\section{Etape 1. \\ Enregistrement d'un produit de PCU spécialisé}

Dans tous les pays, les médicaments et produits de santé doivent être enregistrés et en conformité avec les exigences nationales avant d'être mis sur le marché à travers des réseaux privés ou publics. Le but général de ces exigences réglementaires est d'assurer la sûreté, l'efficacité et la qualité de tous les médicaments délivrés au public. Si un produit n'est pas enregistré (en général suite à l'étude de documents liés à la réglementation ou à des entretiens avec des informateurs clefs), il faut alors suivre le processus d'enregistrement décrit ci-dessous. Si les PCU sont déjà enregistrées dans le pays, passez à l'étape 2, l'introduction des PCU.

Le processus d'enregistrement d'un produit comprend en général les étapes suivantes:

a. Identifier les partenaires pour l'enregistrement des PCU.

b. Mener une évaluation de préenregistrement si nécessaire.

c. Sélectionner les partenaires pour mener un plaidoyer auprès des autorités compétentes.

\section{Identifier les partenaires pour l'enregistrement des PCU}

L'enregistrement des produits pharmaceutiques peut être un processus long et complexe, impliquant plusieurs étapes et conditions. C'est pour cette raison que dans la plupart des pays, les responsables des programmes de santé de la reproduction se sont appuyés sur les fabricants des produits et/ou les distributeurs locaux de produits pharmaceutiques pour qu'ils prennent en charge le processus d'enregistrement et déposent les demandes et documents nécessaires. Ceci présente également l'avantage, en impliquant le secteur commercial, pour obtenir la distribution des PCU dans les pharmacies et les autres points de vente. L'expérience a montré qu'un partenaire local nongouvernemental est en général nécessaire pour mener le processus d'enregistrement. L'agence gouvernementale responsable de l'enregistrement des produits de santé devrait être consultée pour ses conseils sur les procédures d'enregistrement des produits exigées dans le pays.

Les informations couramment exigées pour l'enregistrement des PCU comprennent des informations sur le produit, sur le processus de

\section{Conseils pour le choix du régime}

Il est important de s'assurer qu'au moment de soumettre la candidature pour l'enregistrement, les dernières informations disponibles sur le médicament soient utilisées. Par exemple, l'OMS a approuvé le régime d'une pilule de CU contenant 1.5 mg de levonorgestrel. Il est donc conseillé d'enregistrer d'abord la prise de $1.5 \mathrm{mg}$ de levonorgestrel puisqu'en l'enregistrant après avoir obtenu l'accord pour la prise de deux pilules de $0.75 \mathrm{mg}$ de levonorgestrel chacune, il faudrait recommencer tout le processus.

fabrication, la réglementation pour l'emballage, les résultats des recherches cliniques, des tests de qualité du produit et d'autres informations spécifiques au produit (PATH 2004). La substance active des PCU se trouve également dans les autres contraceptifs disponibles dans la plupart des pays, les agences gouvernementales de régulation peuvent donc ainsi faciliter l'enregistrement des PCU. Le fabricant du produit devrait fournir des informations techniques dans un dossier confidentiel soumis directement à l'agence de régulation.

\section{Les tests sur le terrain en vue de l'enregistrement}

En Ethiopie, l'autorité nationale administrative de contrôle pour les médicaments (national Drug Administration Control Authority) exige que les nouveaux médicaments soient «testés sur le terrain» avant leur enregistrement. Pour le Ministère de la Santé (MS), pour introduire les PCU dans l'ensemble de leurs méthodes, il était nécessaire de mener des recherches dans le pays même. Au lieu de réutiliser les essais cliniques existants et acceptés universellement, le MS a entrepris une étude de recherche opérationnelle. Celle-ci a démontré à la fois la faisabilité de l'offre des PCU dans les établissements gouvernementaux et aussi leur acceptabilité parmi les client(e)s. Combinée avec des données cliniques globales, elle a fourni les bases pour un enregistrement efficace pour les toutes premières PCU spécialisées et enregistrées dans le pays en 2006. (Keesbury, Aytenfisu, et Bradford 2007). 
Mener une évaluation de pré-enregistrement si nécessaire

Un test ou une évaluation peut être ou non un prérequis à l'enregistrement du produit. Si cela est exigé, il est nécessaire d'identifier:

$\checkmark$ L'organisation responsable du test (institution gouvernementale ou ONG).

$\checkmark$ Le type de test (par exemple, un test biomédical ou sur le terrain).

$\checkmark$ Qui est responsable de l'utilisation des résultats lorsqu'ils sont disponibles.

Même lorsqu'une évaluation de pré-introduction n'est pas exigée par le gouvernement, les preuves fournies par une telle évaluation peuvent être utilisées dans des campagnes de plaidoyer ultérieures pour enregistrer et intégrer les PCU dans le programme national de santé de la reproduction, et permettre également d'éviter une éventuelle opposition des groupes politiques et religieux.

Dans certains cas, le programme national de SR ou une ONG peut faciliter le processus d'enregistrement en menant des études de faisabilité, des enquêtes de marché et/ou des sondages sur «la propension à payer» (qui fournit des informations sur la demande, le marché, l'acceptabilité et le prix du produit) et en augmentant l'intérêt des fabricants et des distributeurs, y compris les responsables des programmes de SR et des agences de régulation.

\section{Introduire les PCU avec la méthode Yuzpe}

Il est possible d'introduire les PCU dans les pays n'ayant pas de produits spécialisés enregistrés en utilisant la méthode Yuzpe, qui implique la délivrance de CO combinés déjà enregistrés et utilisés pour la contraception dans la plupart des pays du monde. Dans certains pays d'Amérique latine, les premiers efforts de plaidoyer comprenaient des séminaires et des ateliers nationaux et internationaux pour présenter la méthode Yuzpe et obtenir l'adhésion d'acteurs importants parmi lesquels les responsables politiques et les ONG. En conséquence, la méthode Yuzpe fut intégrée dans les directives officielles pour la prestation de services de planification familiale au Brésil et dans d'autres pays, avant que les produits spécialisés ne soient disponibles (Vernon 1998; Diaz et al. 2007).
Sélectionner les partenaires pour mener un plaidoyer auprès des autorités compétentes

Les chances de succès de faire changer les politiques et d'influencer les décisions concernant les programmes sont plus élevées si un grand nombre d'organisations militent pour le changement. Dans la mesure du possible, les partenaires crédibles tels que les organisations prestataires de services de SR, les organisations féministes et d'aide juridique, les responsables du MS et les donateurs devraient être impliqués dans la promotion de l'introduction des PCU auprès des autorités compétentes. Le plaidoyer sert à sensibiliser les responsables politiques et le public, et peut éventuellement réduire le risque de voir s'exprimer des objections de la part de la communauté.

\section{Etape 2.}

\section{Introduire les PCU dans le secteur public et auprès des ONG}

Dans la plupart des pays, l'enregistrement d'un produit est effectué par les laboratoires et distributeurs pharmaceutiques, la mise en place des PCU dans les pharmacies du secteur privé suit donc de près l'enregistrement du produit. A l'opposé, l'introduction du produit dans le secteur public et les organisations de prestation de service non-gouvernementales requiert en général une promotion intensive et une assistance technique. Dans la plupart des pays d'Amérique latine, au Bangladesh, en Inde, au Népal et au Pakistan par exemple, les PCU étaient enregistrées et disponibles dans le secteur privé plusieurs années avant leur introduction dans les programmes du secteur public. Des expériences dans plusieurs pays montrent que le processus d'introduction des PCU dans le secteur public et/ou le secteur des ONG varie considérablement. Les indications suivantes visent donc principalement à servir de liste de contrôle des problèmes à prendre en compte lors de la planification d'un processus d'introduction. En outre, l'engagement du gouvernement ainsi que son contrôle sur le processus est essentiel pour créer un contexte de politique favorable. Pour y parvenir, il faut souvent des partenaires au développement pour soutenir financièrement le processus d'introduction, élargir la base des intervenants nationaux et/ou développer une stratégie nationale solide que le gouvernement peut approuver. La phase d'introduction peut comprendre les quatre activités suivantes:

$\checkmark$ Mener une évaluation diagnostique pour l'introduction/la réintroduction des PCU.

$\checkmark$ Collaborer avec les intervenants et mener des plaidoyers basés sur la recherche avec les responsables politiques. 
$\checkmark$ Si nécessaire, mener des recherches opérationnelles et des études de faisabilité et/ou d'acceptabilité.

$\checkmark$ Inclure les PCU dans les directives de PF et d'autres documents réglementaires et planifier son introduction en plusieurs étapes.

\section{Mener une évaluation diagnostique pour l'introduction/la réintroduction des PCU}

Les évaluations diagnostiques sont essentielles dans l'étape de pré introduction et devraient inclure des consultations et un dialogue multisectoriels pour identifier les problèmes et sources de résistance éventuels liés aux produits et services de PCU. Les évaluations stratégiques constituent un exercice multipartite et en tant que tel, contribuent à apporter des connaissances, à soutenir le consensus parmi les participants nationaux et à étendre la prise de décision au delà du gouvernement. L'évaluation devrait inclure des consultations avec les experts et les intervenants, ainsi que des études qualitatives et quantitatives pour évaluer les points de vue des décideurs, fournisseurs et utilisateurs. Les sources d'information pour l'évaluation peuvent inclure, entre autres, des enquêtes démographiques et de santé et d'autres recherches nationales et internationales, des documents réglementaires, des informateurs clefs pour la prestation de service, la réglementation et les organisations pour la promotion de la santé de la femme et d'aide juridique. L'évaluation devrait:

Aider les programmes à comprendre les politiques actuelles de planification familiale et l'organisation des services, à faire des recommandations sur les endroits où les PCU devraient être proposées, et quelles personnes pourraient les proposer; comment identifier et former les prestataires; quels seraient les matériels d'IEC nécessaires; et comment inclure les PCU dans le processus d'approvisionnement et de distribution des produits et dans le système d'information et de gestion. L'évaluation devrait également établir si des réglementations ont besoin d'être mises à jour, ainsi que les processus pour les changer, et quelle serait la meilleure manière d'intégrer les PCU dans les directives nationales pour la prestation de services de PF.

\section{Utiliser les recherches pour démontrer l'acceptabilité des PCU.}

Au Bangladesh, les recherches ont montré que 33 pour cent des grossesses étaient non planifiées en raison d'un échec de la méthode ou d'un rapport non protégé. Un grand nombre de ces grossesses ont donné suite à un avortement réalisé dans des conditions à risque et entraînant d'éventuelles complications ou même la mort. Les rapports non protégés sont communs et fréquents. Ces faits soutiennent l'introduction des PCU dans le programme national en tant que méthode de secours pour les méthodes de planification familiale actuelles (Khan and Hossain 2004).

Au Ghana, PPAG affilié à l'IPPF a facilité l'introduction des PCU dans le secteur public en menant une expérience pour démontrer l'acceptabilité des PCU par les utilisateurs, en formant les prestataires, en initiant des activités pour susciter la demande, en soutenant l'enregistrement d'un produit spécialisé, en assurant sa disponibilité, en se le procurant et en le distribuant aux prestataires dans les secteurs publics et privés (Osei 2007).

Au Mexique, l'introduction des PCU dans le secteur public a nécessité d'importants efforts de plaidoyer pendant près d'une dizaine d'années ainsi que l'examen externe des preuves scientifiques montrant que les PCU n'étaient pas abortives. Le soutien des groupes féministes et d'autres ONG, ainsi que des représentants du MS ont fait partie des facteurs qui ont mené à leur introduction (Lara et al.2007).

Le gouvernement du Népal s'est appuyé sur des matériels d'IEC développés au Bangladesh et en Inde et sur l'assistance technique de Population Council et d'une institution nationale de recherche, le Centre Népalais de soins pour la fertilité (Nepal Fertility Care Centre) pour introduire les PCU en plusieurs étapes (Sherestha, Khan et Hossain 2008).

Au Pakistan, les responsables de programmes s'inquiétaient d'une éventuelle réaction violente des musulmans conservateurs lors de l'introduction des PCU. Par conséquent, l'introduction de la méthode n'a débuté qu'après une suite d'études menées par des acteurs clefs, au Bangladesh, un autre pays avec une population à prédominance musulmane conservatrice, pour observer comment elles étaient fournies et sonder la réaction de la population suite à leur introduction (Khan et al. 2008a). 
$\checkmark$ Présenter des données pour défendre l'utilité des PCU pour cette population en décrivant les taux de rapports non protégés sur le plan national, la proportion de grossesses non désirées, l'incidence des avortements à risque et sans risque, ainsi que les taux d'échec de la méthode, entre autres.

$\checkmark$ Identifier les populations spécifiques qui pourraient bénéficier de l'introduction des PCU et la faisabilité d'atteindre ces populations grâce à des systèmes de prestation de service variés. Comme pour la plupart des programmes de SR, les secteurs vulnérables de la population, tels que les jeunes femmes, les pauvres dans les villes et les populations déplacées et réfugiées, sont plus difficiles à atteindre.

$\checkmark$ Rassembler des informations pour aider à comprendre le contexte culturel et les sensibilités autour des PCU, quels groupes pourraient éventuellement s'opposer à son introduction et de même, quels arguments pourraient-ils invoquer et quels types de messages pourraient être envoyés pour informer correctement les décideurs.

$\checkmark$ Identifier les intervenants les plus utiles qui pourraient collaborer avec des programmes de plaidoyer et qui devraient recevoir des informations sur les PCU. Dans plusieurs pays, ceux-ci ont compté non seulement les organisations de prestation de service de santé reproductive mais aussi les organisations pour la santé des femmes, les membres des autorités législative et judiciaire et les médias.

\section{Collaborer avec les intervenants et mener des plaidoyers basés sur la recherche avec les responsables politiques}

Les ONG et les institutions de recherche à travers le monde ont joué un rôle important en encourageant les gouvernements à introduire les PCU dans leurs services de santé de la reproduction. Pour cela, ils ont fourni des informations scientifiques aux responsables qui expliquent les raisons de l'introduction de la CU; ils ont également partagé leurs expériences d'autres programmes. Les ONG jouent aussi habituellement un rôle majeur pour garantir

\section{Se servir des recherches pour augmenter la disponibilité des PCU}

Au Bangladesh, les responsables politiques ont mis en doute l'acceptabilité sociale de la méthode et ont eu besoin de comprendre les changements opérationnels nécessaires pour introduire les PCU dans le système de santé public. Une étude formative suivie par des recherches opérationnelles a montré que les femmes utiliseraient les PCU si elles étaient disponibles, qu'il était possible d'introduire les PCU dans le programme du gouvernement et que les auxiliaires médicaux et les travailleurs communautaires pouvaient fournir des PCU. Ces résultats ont facilité le changement de politique nécessaire ainsi que l'introduction des PCU dans le programme national de planification familiale (Khan et Hossain 2004).

En Inde, les PCU étaient tout d'abord disponibles uniquement sur prescription médicale, ce qui en limitait l'usage. Le corps médical s'est opposé à faire des PCU un médicament en vente libre, ce qui a empêché les auxiliaires médicaux de les fournir. Les responsables politiques ont exigé des recherches pour montrer que les auxiliaires paramédicaux et les agents en contact avec le public pouvaient fournir des services d'une qualité équivalente à celle des médecins. Les résultats de l'étude l'ont prouvé et les responsables politiques ont accepté de changer les réglementations nationales pour rendre les PCU disponibles en vente libre (Kumar et al. 2007).

En Amérique latine, sept pays ont autorisé les PCU après que le MS et les responsables de programmes d'ONG ont assisté à une conférence pour examiner les résultats de projets de recherche opérationnelle dans d'autres pays ainsi que d'autres preuves scientifiques. Ils ont aussi reçu une aide technique pour adapter la méthode à leurs directives et leurs systèmes pour la prestation de services (Vernon 1998).

Malgré près d'une décennie d'offre des PCU dans le secteur public, leur utilisation dans les cliniques et les hôpitaux Kenyans reste limitée. Pour aider le MS à atteindre son double objectif de garantir l'accès des PCU à la fois aux adolescents et aux victimes d'agression sexuelle, Population Council a mené une évaluation nationale des fournisseurs et établissements du secteur public. Celle-ci a montré que les services de PCU pour les victimes d'agression sexuelle étaient biaisés et que la majorité des prestataires n'étaient pas suffisamment formés pour offrir les PCU dans le cadre de la planification familiale. La réaction du MS a été de s'employer à renforcer les formations sur les PCU avant le service dans les écoles médicales et d'infirmiers et de proposer des mises à jour sur la technologie des contraceptifs aux prestataires en service (Keesbury, Owino, et Bradford 2007). 
la disponibilité des stocks de CU pendant les projets pilotes d'introduction, convoquer les intervenants, développer des programmes de formation et dispenser la formation, aider à l'élaboration des directives sur les PCU et mener des évaluations diagnostiques. Les chances d'introduire et de vulgariser les PCU dans le secteur public sont plus élevées dans le cadre d'un partenariat avec des organisations influentes auprès des autorités gouvernementales, qui sont connues pour être honnêtes et observer une rigueur scientifique dans leur travail. En l'absence d'un engagement fort du secteur public, les PCU pourraient devenir la responsabilité des ONG ou du secteur privé uniquement. Comme les premiers ont en général une couverture limitée et que le second répond aux lois du marché, cela voudrait dire que les PCU ne seraient pas disponibles à un niveau national, ni pour ceux qui ont des besoins spécifiques, comme c'est le cas au Nigéria (Apsel 2007).

\section{Si nécessaire, mener des recherches opérationnelles et des études de faisabilité et/ou d'acceptabilité}

Dans plusieurs pays en Asie, Afrique, Amérique latine et Caraïbes, un diagnostic de la situation, des recherches opérationnelles et de sciences sociales ont été entrepris pour fournir aux responsables politiques la preuve de la faisabilité et de l'acceptabilité des PCU. Sans ces preuves, cela n'aurait pas été possible de revoir les politiques actuelles, de concevoir une stratégie d'introduction ou de garantir que l'introduction des PCU ne conduise pas à des controverses ou à des réactions violentes. Lorsque les résultats des études de faisabilité, de recherches opérationnelles et d'expériences de programmes des pays voisins sont concluants et crédibles, il n'est peut être pas nécessaire de mener des études supplémentaires.

\section{Contenu d'une session de formation classique}

Première session: Résumé de la situation démographique et de santé de la reproduction et discussion du rôle de la CU en tant qu'intervention de santé de la reproduction.

Deuxième session: Définition de la $\mathrm{CU}$ et discussion sur quand et comment les PCU devraient être prises.

Troisième session: Directives pour la prestation de services sur comment délivrer les PCU en fonction des besoins spécifiques du client.

Quatrième session: Le conseil pour les PCU, la gestion du suivi et de la logistique et foire aux questions.

Exemple du Bangladesh (Khan et Hossain 2008)

\section{Inclure les PCU dans les directives de PF et d'autres documents réglementaires et planifier son introduction en plusieurs étapes}

L'introduction des PCU dans les directives pour la prestation de service est un aspect important du processus d'introduction, surtout si l'on veut généraliser la méthode. Ce processus peut comporter une période de plaidoyer, d'assistance technique et une planification stratégique de la part des intervenants et des experts de la santé de la reproduction. Dans certains pays comme le Mexique, les nouveaux contraceptifs ne peuvent pas être introduits dans les programmes du secteur public avant d'avoir été intégrés dans les directives nationales pour la prestation de service

\section{La formation classique}

En Zambie, une formation classique a été dispensée pour donner aux officiers de police les compétences pour offrir les PCU aux victimes d'agression sexuelle. En tant que personnel non médical, leur formation a nécessité plus de temps que pour les prestataires de soins de santé. Cette formation a été dispensée sur 5 jours et suivait une version condensée du curriculum du MS pour les distributeurs communautaires et ceux sur les lieux de travail. Elle a insisté sur les points essentiels concernant la physiologie de la reproduction, les technologies contraceptives, la réponse en cas d'agression sexuelle, l'intégration avec les autres services et les conseils aux clients/victimes (Keesbury, Aytenfisu, et Bradford 2007).

Au Bangladesh, une formation indépendante a été dispensée pour former tout le corps des prestataires de planification familiale (plus de 45000 prestataires). Des responsables de programmes seniors et des représentants du district ont été également formés sur les PCU avant la formation des formateurs et des prestataires de service. Au départ, un groupe de maîtres formateurs a été constitué pour former un grand nombre de formateurs et la formation indépendante a ensuite été étendue en utilisant une démarche en cascade (voir page opposée) (Khan et Hossain 2008). 
et que le changement ait été rendu officiel par sa parution dans le journal officiel. (Dans le secteur privé cependant, les réglementations habituelles pour l'enregistrement des médicaments sont appliquées). Comme cela fut le cas au Bangladesh et au Kenya, si le personnel de l'organisation menant les activités d'introduction peut collaborer étroitement avec le gouvernement (comme par exemple en faisant partie des comités techniques), il est possible de faire des changements au niveau des directives et des programmes d'enseignement.

\section{Etape 3.}

\section{Former et renforcer les capacités des fournisseurs de PCU}

La formation des prestataires de service est une composante cruciale de l'introduction et de la généralisation des services de PCU. Dans bien des situations, surtout dans les grands pays, la formation peut être coûteuse et problématique sur le plan logistique. La section suivante propose des stratégies de formation variées qui peuvent être choisies selon les besoins et le contexte, et qui aideront à renforcer les capacités globales du système de SR pour organiser et suivre la formation sur l'introduction des PCU.

$\checkmark$ Qui devrait être formé

$\checkmark$ Quelles informations un prestataire devrait-il connaître

$\checkmark$ Les stratégies de formation des prestataires de service

$\checkmark$ Les stratégies pour généraliser la formation sur les PCU

\section{Qui devrait être formé ?}

Les fournisseurs des PCU varient selon les pays, cela dépend de si elles sont proposées par les secteurs public ou privé, par les cliniques, les

\section{Des matériels de formation innovants}

Au Venezuela, ProSalud InterAmericana, une ONG nationale a créé un $C D$ interactif qui est distribué gratuitement aux prestataires de service pour leur auto-formation sur les PCU. Le CD a été utilisé par les ONG et le MS dans plusieurs pays dans la région (Martin 2004). Pour une présentation du $C D$, voir http://www.prosaludinteramericana.org/esp/cd/ demo.html

Le comité central pour la santé en Zambie, avec ses ONG partenaires a développé un guide d'autoformation sur «La planification familiale en santé de la reproduction, » qui a inclus un module sur les PCU et des conseils. Les tests réalisés avec cet outil ont montré qu'il était aussi complet qu'une formation en classe, les prestataires avaient toutefois besoin de 10 à 15 semaines pour achever la formation à leur propre rythme (Keesbury 2007).

pharmacies, les travailleurs de proximité ou le personnel non médical, de la réglementation, du contexte culturel et des préférences de la population principalement ciblée. Dans les secteurs à la fois public et privé, les personnes suivantes ont contribué à fournir des informations et des services de CU aux femmes (PATH 2004):

$\checkmark$ Les infirmiers et les sages-femmes

$\checkmark$ Les docteurs

$\checkmark$ Les auxiliaires médicaux

Les agents de santé communautaires formés

$\checkmark$ Les adolescent(e)s pair-éducateurs

$\checkmark$ Les pharmaciens et le personnel des pharmacies du secteur privé

Les conseillers pour la violence

\section{La formation intégrée}

En Inde, le MS a adopté une démarche intégrée pour former 200000 agents de santé sur les PCU en raison du coût prohibitif de l'organisation de formations specifiques La formation pour les PCU a été intégrée au programme de formation pour la santé de la reproduction et de l'enfant dans le cadre duquel les auxiliaires paramédicaux sont formés pendant trois jours. Tous les auxiliaires paramédicaux ont ensuite été formés à ce nouveau service, pendant une session de deux heures sur les PCU. Par la suite, les PCU ont été incorporées au guide du MS de mise à jour sur la technologie contraceptive (MJTC).

La méthodologie pour la formation intégrée a également été adoptée avec succès au Guatemala, au Salvador et au Kenya. Au Kenya, les services de PCU ont été renforcés et sont des modules réguliers des formations nationales de MJTC dispensées par le Ministère de la Santé. Cette stratégie a pu garantir que les PCU étaient considérées comme un élément essentiel des services habituels de planification familiale et de santé de la reproduction et non comme un fardeau supplémentaire pour les prestataires de service. 
sexospécifique/les victimes de viol et l'aide juridique

$\checkmark$ La police et le personnel médico-légal

\section{Quelles informations un prestataire devrait-il connaître}

Un curriculum de formation devrait inclure les informations essentielles sur les PCU, une mise à jour sur les autres méthodes contraceptives, les problèmes de santé de la reproduction liés tels que les IST/VIH et le conseil sur l'importance de faire la transition vers une contraception régulière et l'offre/ l'orientation vers des services (PATH 2004). Les prestataires de santé des cliniques et des communautés devraient également être formés pour inclure les PCU dans toutes les sessions de conseil sur la PF et les activités d'éducation à la santé. Le contenu, les sujets principaux et le type d'informations communiquées pendant la formation pourront varier en fonction du type de prestataire. Par exemple, le personnel de PF pourrait avoir besoin d'informations complémentaires sur la transition vers d'autres services de PF et les IST, tandis que les pharmaciens pourraient bénéficier de conseils sur la fourniture d'un service confidentiel. Le renforcement des capacités peut même débuter avant l'enregistrement d'une PCU spécialisée puisque les COC sont disponibles dans la plupart des pays et peuvent être utilisés pour la méthode Yuzpe avant l'introduction d'une PCU spécifique.

\section{Les stratégies de formation des prestataires de service}

$\checkmark$ Formation ciblée. Former spécifiquement sur les PCU est un moyen efficace de renforcer les capacités d'un groupe spécifique de prestataires afin qu'ils aient une compréhension globale des PCU. Ce type de formation peut en général être dispensé pendant une courte période de temps, dans une classe, pour les prestataires qui ont déjà une compréhension et des compétences de base dans la prestation de service de PF. Malheureusement, cela peut s'avérer coûteux, puisqu'un budget

\section{Etapes de la formation en cascade dans le secteur public au Bangladesh}

- Former un groupe de maîtres formateurs qui, ensuite...

$\checkmark$ Forment un groupe de formateurs qui ensuite...

$\checkmark$ Forment les prestataires de service

\section{Intégrer les PCU dans la formation avant le service}

Au Kenya, les défenseurs des PCU ont aidé à revoir le curriculum pour la planification familiale du National Medical Training College, en fournissant les ressources et l'expertise technique nécessaires pour renforcer les instructions sur les PCU. Population Council soutient cet effort en dispensant un cours de révision sur la CU pour les tuteurs dans tout le pays.

Au Bangladesh, un chapitre sur les PCU a été introduit dans le guide national de la contraception, le livre de référence pour tous les auxiliaires médicaux et professionnels de la santé travaillant dans le secteur de la planification familiale.

En Inde, les PCU ont été incorporées de la même façon à tous les manuels de formation du Ministère de la Santé et du Bien être Familial, comme les mises à jour sur la technologie contraceptive et un guide pour introduire des cliniques de santé reproductive et sexuelle adaptées aux adolescent(e)s.

distinct est nécessaire et ce n'est peutêtre pas la meilleure solution pour les prestataires qui ne peuvent pas s'absenter facilement de leur lieu de travail. Cette stratégie n'est peut-être pas adaptée pour développer à grande échelle des programmes de PCU dans le système public national de santé à moins que le financement nécessaire ne soit disponible.

$\checkmark$ La formation intégrée. La formation sur les PCU peut être intégrée à d'autres formations, telles que les mises à jour sur la technologie contraceptive ou d'autres formations sur les sujets de SR. Cette stratégie peut être plus rentable qu'une formation specifique sur la CU et peut toucher un grand nombre de personnes mais il est important de s'assurer que le contenu et la qualité de la formation ne soient pas compromis. La principale contrainte est qu'elle ne peut être dispensée que s'il existe d'autres formations au moment opportun et appropriées au type de prestataire.

$\checkmark$ L'auto-formation. L'auto-formation est un outil utilisé pour l'enseignement à distance dispensé complètement ou partiellement en dehors d'un 
établissement. Cela exige un encadrement mais peut s'avérer être une solution efficace dans le cas où les ressources pour une formation classique ou intégrée sont inadaptées ou lorsque les prestataires ne peuvent pas s'absenter de leur lieu de travail.

\section{Les stratégies pour généraliser la formation sur les PCU}

Les trois stratégies décrites ci-dessus peuvent s'appliquer lorsque l'on introduit les PCU et peuvent être développées, testées et modifiées grâce à des tests d'introduction pilotes. Lorsque les PCU ont été introduites avec succès dans un programme national, en général à petite échelle, une stratégie de formation différente est normalement nécessaire pour généraliser les PCU dans un système de SR national. Trois démarches peuvent être préconisées:

Une formation en cascade pendant le service. Une formation en cascade peut être un moyen rentable de toucher un grand nombre de prestataires avec une formation pendant le service. Elle peut être utilisée avec le curriculum d'une formation intégrée ou indépendante, en fonction de la disponibilité du financement pour soutenir un programme de formation spécifique pour les PCU (comme cela fut le cas au Bangladesh, avec un financement du gouvernement, de UNFPA et USAID). Il peut également y avoir des programmes de formation réguliers (par exemple, les programmes de MJTC et de santé de la reproduction et de l'enfant en Inde) dans lesquels les formations pour les PCU peuvent être intégrées.

Lors de la conception d'une stratégie de formation en cascade pour un programme destiné à un large public, il est préférable de développer un corps de maîtres formateurs qui peuvent ensuite former les formateurs eux-mêmes. Les maîtres formateurs et les formateurs sont ensuite disponibles pour renforcer les capacités des prestataires de service du système. Au Bangladesh, au total, 300 maîtres formateurs et 2300 formateurs ont été employés à renforcer les capacités de 45000 prestataires de service (Khan et Hossain 2008). La stratégie de formation en cascade dépend du financement disponible, de la motivation et de la disponibilité de tous les groupes de formateurs et d'un niveau constant d'encadrement pour garantir que la formation à tous les niveaux soit de qualité et se déroule tel que prévu.

\section{Des ressources conseils sur les PCU}

Consortium international pour la contraception d'urgence:

$\checkmark$ Matériels pour les clients de CU http://www. cecinfo.org/publications/PDFs/materialsClients/ PATH_client_french_letter.pdf

$\checkmark$ Déclaration de politique (juillet 2003) Mise à jour sur le régime: Dosage et posologie http://www. cecinfo.org/publications/PDFs/policy/Dosage_ Timing_French.pdf

$\checkmark$ Déclaration de politique (juillet 2003) Utilisation répétée de la contraception d'urgence: les données http://www.cecinfo.org/publications/PDFs/ policy/RepeatedUse_French.pdf

$\checkmark$ Déclaration de politique (juillet 2003) Améliorer I'accès à la contraception d'urgence http:// www.cecinfo.org/publications/PDFs/policy/ ImprovingAccess_EC_French.pdf

$\checkmark$ Déclaration de politique (juillet 2003) Contraception d'urgence et avortement médical http://www.cecinfo.org/publications/PDFs/policy/ EC_MedicalAbortion_French.pdf

Pathfinder:

$\checkmark$ Emergency Contraceptive Pills, 2nd edition (2007) http://www.pathfind.org/site/DocServer/ECP2ed_for_ web.pdf?docID $=10203$

PATH :

$\checkmark$ Resources for Emergency Contraceptive Pill Programming: A Toolkit. 2004. www.path.org/files/ RH_ec_toolkit.pdf

La formation sur la CU avant le service par des organismes de formation.

La manière la plus rentable pour généraliser les PCU, sur le long terme en tout cas, est de les intégrer aux formations de base pour le personnel susceptible de les offrir pendant leur travail. L'incorporation des PCU dans les manuels et programmes de formation des organismes de formation médicale (comme les facultes de médecine, de pharmacie, les écoles d'infirmiers et les cours pour les auxiliaires médicaux) est cruciale pour institutionnaliser la formation sur les PCU et garantir sa durabilité dans le système de santé. Cette démarche garantira également que la formation sur les PCU est dispensée aux professionnels de la santé travaillant à la fois dans le secteur public et dans le privé.

$\checkmark$ Assurance qualité et formation de révision. Le suivi de la formation est important pour garantir une assurance qualité constante. Des cours de révision qui peuvent être dispensés lors des visites de supervision de routine, de 


\section{Les stratégies pour impliquer les médias}

ECafrique a énormément travaillé avec des partenaires médiatiques pour changer le discours public sur la CU. Conjointement avec un groupe de médias Kenyan, il a préparé un kit de «réponse rapide» pour aider les journalistes et les ONG à répondre aux reportages erronés des médias et à fournir des articles défendant la CU et il a appuyé un atelier de renforcement des capacités de quatre jours pour 22 journalistes éminents de la santé d'Afrique australe et orientale. L'atelier a donné suite à 50 histoires sur la CU et d'autres sujets liés à la SR, relayés dans la presse, à la radio, la télévision et dans d'autres médias vernaculaires (ECafrique 2006).

Au Mexique, un kit destiné à la presse a été envoyé aux organisations de médias qui traitaient des sujets de santé. Par la suite, plus de 21 articles ont été publiés dans les magazines et les journaux nationaux, six rubriques ont été diffusées à la radio, et une mention a été faite lors d'un programme d'information national; les PCU ont également été incluses dans le scénario d'un feuilleton télévisuel. On a estimé que ce simple matériel a permis de toucher 11.8 millions de personnes pour un coût total inférieur à 1000 US (Givaudan, Fuertes, et Vernon 1998).

En Inde et au Bangladesh les médias ont également joué un rôle positif grâce à la publication d'articles soulignant et soutenant le besoin d'introduire les PCU dans le programme national de santé de la reproduction. En Inde, une couverture médiatique importante faisant suite à un projet de plaidoyer soutenu par la Fondation Packard, a joué un rôle déterminant dans l'adoption ultérieure des PCU (Khan et Hossain 2008).

La presse Argentine a couvert l'annonce de l'offre gratuite des PCU par le gouvernement et a soutenu leur introduction dans les services de santé universitaires. Les journaux ont également dénoncé les difficultés juridiques, arguant que la nouvelle réglementation affectait les plus vulnérables qui ne pouvaient pas se permettre d'acheter les PCU en pharmacie (La Nación 2007). Les médias se sont largement engagés dans le débat public sur l'offre au public au Chili, prenant position pour, contre ou restant neutre face à la CU (La Nación 2006 ; La Nación 2007; La Nación 2008; Faúndes et al. 2007).

suivi et/ou d'assurance qualité par des superviseurs, devraient revoir et insister sur les éléments clefs pour l'offre des PCU et également actualiser les connaissances des prestataires sur les modifications ainsi que les progrès récents des directives pour la prestation de service. Une attention particulière devrait être portée pour s'assurer que les opinions personnelles des prestataires ne les empêchent pas de donner des informations, ni de proposer des conseils appropriés, qu'ils insistent sur la nécessité de faire la transition vers une contraception régulière et qu'ils recherchent les autres besoins en SR, en particulier la prise en charge des IST/VIH. Au Bangladesh par exemple, tous les superviseurs ont été formés à poser des questions sur les services de PCU pendant les visites et à engager des discussions de 15 minutes pendant les réunions mensuelles avec le personnel. L'offre des PCU est maintenant incluse dans le système d'information et de gestion (SIG) du ministère.

\section{Etape 4. Défendre les PCU et sensibiliser le public}

Sensibiliser le public aux PCU est une étape critique de l'introduction pour s'assurer que les populations visées, générales et groupes spécifiques, sont sensibilisées sur la méthode, qu'elles savent comment elle agit, et où elles peuvent se procurer des PCU. Les messages clefs et les moyens de communication sont décrits cidessous pour plus de détails et de conseils (voir l'encadré).

L'expérience a montré que les éléments suivants seront en général nécessaires pour s'assurer que les utilisateurs potentiels connaissent parfaitement les PCU, que les opposants éventuels peuvent être informés sur les bénéfices des PCU et que l'on peut défendre l'intégration des PCU dans le programme national de SR.

$\checkmark$ Comprendre le contexte social, culturel, religieux et juridique.

$\checkmark$ Choisir des canaux de communication en fonction de leur audience.

$\checkmark$ Utiliser les médias pour toucher des publics spécifiques.

$\checkmark$ Développer les messages clefs. 


\section{Comprendre le contexte social, culturel, religieux et juridique}

Les réglementations juridiques du pays stipulant où et comment les PCU peuvent être promues doivent être bien comprises. De nombreux pays n'autorisent pas les messages sur la contraception dans les médias ou restreignent leur contenu ainsi que le moment de leur diffusion. Par exemple, de nombreux pays musulmans interdisent la publicité pour les méthodes de planification familiale en général et pour le préservatif en particulier. Il est également essentiel de comprendre l'environnement social, religieux, culturel et politique du pays pour éviter de créer d'inutiles controverses.

Les PCU sont relativement plus faciles à introduire et à généraliser dans des contextes ayant des niveaux plus élevés de connaissance et d'utilisation des contraceptifs. Toutefois, lorsqu'un grand nombre de couples sont déjà protégés par une méthode contraceptive fiable, l'impact éventuel d'une introduction réussie des PCU peut sembler marginal aux décideurs. Dans ce cas, la justification pour l'introduction des PCU dans un pays peut être donnée en termes de besoin pour les couples faisant face à un échec de la méthode ainsi que leur rôle dans les soins suite à un viol. Au Bangladesh où 60 pour cent de la population utilise la contraception, un tiers des grossesses ne sont pas planifiées et cela est en général dû à un échec de la méthode. Des arguments similaires ont été pertinents au Népal et au Pakistan.

La connaissance et la sensibilisation aux PCU parmi les prestataires ainsi que les clients potentiels dans toutes les régions du monde sont toujours très faibles. Par conséquent, les campagnes médiatiques pour sensibiliser rapidement et corriger les connaissances sur les PCU au niveau de la population peut faire augmenter rapidement et de manière significative l'utilisation des PCU. Le succès des campagnes médiatiques, comme en Inde, au Kenya et dans plusieurs pays d'Amérique latine a été démontré par la forte augmentation des ventes de PCU dans les zones urbaines après leur lancement. Des périodes courtes de grande exposition à des informations appropriées peuvent être aussi efficaces que des campagnes médiatiques de longue durée qui ciblent des populations spécifiques ou géographiquement limitées.

\section{Choisir des canaux de communication en fonction de leur audience}

Les deux audiences principales des campagnes médiatiques sont les utilisateurs potentiels des PCU et les personnes qui peuvent en faire la promotion et fournir des informations, des conseils et des services. L'évaluation des médias disponibles dans le pays, y compris leurs politiques en matière de publicité pour les produits contraceptifs et le type d'audiences qu'ils touchent est essentielle avant de décider d'utiliser ce canal.

Il est possible de toucher des audiences variées et de communiquer des messages différents en fonction du média utilisé (la presse, la radio ou une combinaison des deux). Une évaluation peut être menée en considérant les médias d'informations suivants et leurs potentialités:

$\checkmark$ Les journaux, les magazines, les stations de radio et les chaînes de télévision publient des informations sur les méthodes contraceptives, la santé de la reproduction, le genre et l'autonomisation des femmes. Au Bangladesh par exemple, les journalistes reçoivent des informations sur les PCU et écrivent des articles pendant la période de pré-introduction. En Inde, les laboratoires pharmaceutiques utilisent les publicités à la télévision pour sensibiliser le public aux PCU.

$\checkmark$ Les agences de publicité et de communication peuvent permettre de déterminer les canaux, le moment, la fréquence et la durée de la campagne prévue. Elles peuvent aussi aider à

\section{Réagir face à des médias défavorables}

Au Kenya et au Botswana, des reportages dans les médias ont suggéré qu'un accès accru à la CU via les pharmacies encourageait les adolescent(e)s à «prendre les PCU comme du chocolat» (Okwemba et Anyona 2004). Ces reportages, fondés essentiellement sur des anecdotes provenant d'un petit nombre de pharmaciens, ont réellement compromis l'offre des PCU dans les deux pays. En réaction à ces articles, les responsables politiques ont craint qu'une disponibilité accrue des PCU n'entraîne des comportements sexuels à risque dans un contexte de prévalence du VIH élevée. Les défenseurs des PCU dans les deux pays ont réussi à faire pression contre les restrictions à leur accès grâce à une communication personnelle et ces deux Gouvernements travaillent maintenant avec ECafrique pour développer des stratégies pouvant garantir de manière responsable la fourniture de PCU dans les établissements des secteurs public et privé. 
développer et délivrer les messages de campagne. Dans certains pays comme le Mexique, ces agences ont accepté de travailler à titre gracieux. Dans d'autres comme le Kenya, le Ghana et l'Ethiopie, des sociétés de marketing social ont lancé des campagnes médiatiques visant à augmenter les ventes dans le secteur privé principalement.

$\checkmark$ Les producteurs et les scénaristes de feuilleton et d'autres programmes de divertissement pourraient intégrer la CU dans leur scénario. Au Mexique, un kit destiné à la presse a été envoyé à plusieurs sociétés de médias avec des conseils sur l'utilisation du matériel fondés sur les exemples d'autres pays. Les scénaristes de l'un des feuilletons les plus populaires de l'époque (Mijada de Mujer [Le regard d'une femme]) ont introduit les PCU dans le scénario du programme suivant l'exemple d'une insertion similaire dans la série «ER » (médicale urgences), populaire aux EtatsUnis.

$\checkmark$ Des canaux communautaires, comme les radios et les théâtres communautaires et les groupes de jeunes, pourraient éventuellement inclure les PCU dans leurs activités.

$\checkmark$ Les lignes d'assistance téléphoniques et sur internet, pour la santé et la planification familiale, peuvent également fournir des informations sur les PCU. Au Kenya, les conseillers travaillant pour les lignes d'assistance téléphoniques pour les informations sur les IST/VIH ont été formés à répondre à des questions sur les PCU et à orienter vers d'autres méthodes ou services tels le CDV ou les soins suite à un viol. Au Bangladesh, les laboratoires pharmaceutiques ont utilisé les lignes d'assistance téléphoniques pour répondre aux questions concernant leurs produits de PCU et la façon de les utiliser.

$\checkmark$ Le publipostage: brochures, articles scientifiques, posters et d'autres matériels imprimés peuvent être envoyés au public visé comme les médecins privés, les pharmaciens, les organisations pour la santé des femmes, les écoles et universités, les usines de fabrication et de montage, les centres de soins pour les victimes de viol, entre autres. Au Mexique cette stratégie s'est avérée efficace pour augmenter les connaissances des PCU au sein de ces groupes. De plus, il a été prouvé que les personnes qui reçoivent des matériels d'IEC sont plus susceptibles de les utiliser et d'informer et de former d'autres personnes (Givaudan, Fuertes, et Vernon 1997; Vernon et Llaguno 2001; Martin 2004).

\section{Utiliser les médias pour toucher des publics spécifiques}

Au Paraguay, à travers une alliance avec PSI, la structure locale affiliée à l'IPPF, Promesa a mené un marketing social pour le Pronta ${ }^{\circledR}$, une PCU spécialisée à progestatif seul. Au début, d'importantes recherches ont été menées pour développer la marque et la stratégie marketing, des efforts marketing supplémentaires ont ciblé principalement les prestataires de service et les jeunes. Promesa et PSI ont créé un CD interactif de matériels de formation pour les prestataires. Promesa a également communiqué avec les jeunes par le biais d'un programme radio hebdomadaire, un réseau de pair éducateurs/ promoteurs et d'autres moyens via l'internet et les textos des téléphones portables. Les femmes et hommes mariés ont aussi été touchés grâce à l'intégration des messages sur les PCU en général, ainsi que les informations sur Pronta ${ }^{\circledR}$ en particulier, dans les activités de santé sexuelle de Promesa (Martin 2004).

En l'absence d'une campagne de publicité, une augmentation marquée de la sensibilisation peut avoir lieu lorsque les débats nationaux génèrent spontanément une couverture médiatique, surtout lorsqu'une telle exposition a lieu grâce à des stratégies de communication publiques coordonnées. Cela a été le cas en Argentine, au Chili, en Colombie et au Mexique (La Nación 2007; La Nación 2008).

Dans certains pays, la couverture médiatique a été obtenue par des stratégies de relations publiques soigneusement planifiées et qui incluaient plusieurs éléments comme les conférences de presse, les communiqués de presse, et des kits destinés aux médias largement diffusés. Dans d'autres cas, l'attention médiatique a été obtenue par l'achat de plages radiophoniques par des campagnes marketing à grande échelle ou de marketing social, comme cela a été le cas pour la promotion de Postinor par PSI au Venezuela (ICEC 2005).

\section{Développer les messages clefs}

Des messages différents doivent être envisagés pour chacun des publics lors de la sensibilisation aux PCU. Le cœur du message peut varier en fonction du public mais tous les messages devraient contenir des informations correctes et scientifiquement fondées.

\footnotetext{
$\checkmark$ Pour les utilisateurs et prestataires potentiels. Il est important qu'ils
} 
sachent quelles options de PCU sont disponibles, comment et quand les PCU peuvent être utilisées, la dose et le moment d'administration, les sources d'approvisionnement, le mode d'action, les effets secondaires possibles et leur traitement ainsi que le besoin de faire la transition vers une méthode régulière efficace.

$\checkmark$ Pour les opposants aux PCU. Il est important de communiquer sur le mode d'action des PCU (par exemple sur le fait qu'elles ne sont pas abortives), sur le fait qu'elles n'entraînent pas une augmentation de l'activité sexuelle ou des comportements à risque et qu'elles jouent un rôle de transition en incitant à l'utilisation régulière de méthodes contraceptives plus fiables.

Les étapes suivantes peuvent aider à développer des messages efficaces pour ces deux groupes:

$\checkmark$ Identifier des matériels existants d'IEC sur les PCU qui pourraient être adaptés et utilisés dans les campagnes d'éducation.

$\checkmark$ Pré-tester la clarté des messages, leur sensibilité culturelle et leur justesse et s'assurer qu'ils répondent judicieusement aux besoins d'information des utilisateurs potentiels, des prestataires et des opposants.

$\checkmark$ Envoyer un à trois messages à la fois seulement.

\section{Etape 5.}

Généralisation: Développement à grande échelle et institutionnalisation durable des services de PCU

L'introduction des PCU dans un programme de SR national est en général mise en œuvre sous la forme d'un projet pilote. Une fois que l'on a démontré que l'offre est possible, les défis à relever consistent à: (1) généraliser la fourniture des PCU en développant à l'échelle nationale les pratiques démontrées comme faisables et efficaces pendant la période d'essai; (2) s'assurer qu'elles peuvent être durables sur une base régulière afin que les PCU deviennent une méthode standardisée dans l'ensemble des services disponibles. L'anticipation et la planification pour le développement à grande échelle ainsi que la durabilité devraient être incluses au début de la planification, sinon les activités proposées pourraient ne pas être transposables à grande échelle ni durables sur le long terme. C'est un problème crucial et particulièrement pertinent pour l'introduction des PCU étant données les sensibilités concernant la méthode, même après qu'elle ait fait les preuves que sa fourniture était socialement acceptable et faisable. En Zambie, par exemple, malgré le succès de la phase pilote et de l'introduction par la suite dans les directives nationales de PF, les PCU sont toujours indisponibles dans le secteur public en raison des financements limités pour l'approvisionnement en contraceptifs. Des barrières similaires se sont élevées au Ghana, en Ethiopie, au Nigeria, où les avancées obtenues par les efforts d'introduction ont diminué lors de la phase de généralisation (Muteshi et Keesbury 2007).

Il n'existe pas de formule ni de cadre simple pour généraliser les PCU que l'on pourrait détailler ici, car chaque situation détermine le meilleur moyen de développer un essai réussi à grande échelle. Les leçons clefs tirées d'un ensemble d'expériences pour généraliser les PCU fournissent des conseils sur certains problèmes qui devraient être pris en considération, avant même de démarrer un projet pilote.

1. Le développement à grande échelle de la fourniture des PCU devrait faire partie des objectifs du test d'introduction. Lors de la conception de la phase d'introduction, il faudrait toujours garder à l'esprit que quel que soit le modèle développé pour fournir les PCU à petite échelle, il devrait être également adaptable à la fourniture de la méthode à l'échelle nationale. Que la généralisation se fasse dans le secteur public, privé ou des ONG, cet objectif influera sur les décisions suivantes:

$\checkmark$ Le choix du système de fourniture ou de programme pour offrir la méthode.

$\checkmark$ La conception du modèle de fourniture des PCU (c'est-à-dire qui, où, quel produit, quels messages d'information).

$\checkmark$ Le financement pour l'approvisionnement en produits et les coûts pour la fourniture pendant et après la phase d'essai.

2. L'intervention test devrait répondre à un besoin ressenti pour le programme national et avoir le potentiel pour répondre aux besoins d'une population très importante. Par exemple, réduire le nombre de grossesses non désirées et leurs conséquences négatives est une priorité pour la plupart des programmes de santé (y compris celles dues à un échec de la méthode). Au Bangladesh, environ un tiers des grossesses chaque 


\section{Une démarche par étape pour introduire et développer les PCU à grande échelle: l'expérience du Bangladesh}

1. Etudes diagnostiques: Evaluation des besoins qualitatifs pour les services de PCU

2. Recherche opérationnelle pour évaluer l'acceptabilité des PCU et déterminer les mécanismes opérationnels pour introduire les PCU

3. Recommander les stratégies appropriées au MS en se basant sur les résultats de la recherche opérationnelle

4. Présentation des résultats de la recherche opérationnelle à un niveau national aux médias, responsables de programmes, chercheurs, pédagogues et représentants des ONG

5. Décisions en matière de réglementation pour introduire les PCU dans le programme national de PF en deux étapes

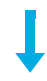

6. Etape I: Introduction des PCU dans l'arrondissement de Dhaka couvrant 45 millions de personnes

7. Développement des matériels d'IEC et de formation

8. Formation de 14007 prestataires de service et prestations de service $\mathrm{s}$

9. Suivi de la Phase I développée à grande échelle

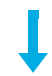

10. Etape II: expansion dans les arrondissements restants, couvrant 89 millions de personnes

11. Poursuivre le suivi et la centralisation des réactions des clients sur la prestation de services

12. Prendre les mesures nécessaires pour améliorer la prestation de services et informer les clients

année ne sont pas prévues et l'on pense que la plupart sont interrompues par un avortement à risque. Les dirigeants politiques et les responsables de programmes ont insisté sur la fourniture de méthodes pour réduire le nombre de grossesses non prévues. Au Kenya, par contre, les responsables politiques s'inquiétaient de ne pouvoir répondre aux besoins en santé de la reproduction de la grande population adolescente du pays. En ce basant sur les recherches montrant que les adolescent(e)s étaient les utilisateurs les plus fréquents des PCU, le MS a décidé de renforcer la fourniture de PCU à cette population cible (Morgan 2007).

\section{La fourniture des PCU dans le} programme national ne requiert pas de grands changements du système en place. La perspective de devoir effectuer des changements importants dans le système d'offre de PF existant suscite en général une forte réticence de la part des dirigeants politiques et des responsables de programmes, ce qui limite l'éventualité d'un développement à grande échelle. Les PCU devraient donc être introduites comme une option supplémentaire au sein du système existant d'offre des contraceptifs. Par exemple, lors de l'introduction au Bangladesh, un formulaire d'une page d'enregistrement pour les client(e)s a été donné à remplir aux prestataires. Après six mois, ce formulaire a été abandonné en raison du travail supplémentaire induit pour les prestataires; ils ont suggéré d'ajouter à la place une colonne dans leur registre de clientèle existant et cet ajustement a été mis en place pendant le développement national à grande échelle.

4. Les responsables de programme en charge du développement à grande échelle de l'offre des PCU devraient être impliqués dans la planification et la mise en ouvre de la phase d'essai. Lorsque la phase d'essai s'est avérée acceptable et faisable, il est plus facile de convaincre les personnes qui auront la responsabilité de la prise de décision pour la planification et le financement de la généralisation des PCU, s’ils connaissent bien et soutiennent le modèle d'introduction mis à l'essai. Si les responsables de programmes n'ont pas le temps de s'impliquer personnellement, ils doivent être régulièrement informés et encouragés à proposer leurs idées ou un soutien administratif. En Ethiopie, le renouvellement au sein du MS a entravé les efforts pour la généralisation car les défenseurs des PCU ont été remplacés par des agents moins en faveur du projet. Ceci a paralysé la généralisation dans le secteur public bien que l'enregistrement des produits réalisé pendant la période d'essai ait permis l'expansion des services pour les PCU dans le secteur privé du pays.

5. Etablir des partenariats stratégiques avec des organisations qui peuvent faciliter la généralisation. En fonction des capacités et des ressources de l'organisation qui soutient les processus 
d'introduction et de généralisation, des partenariats avec des organismes qui ont une expertise technique spécifique et une crédibilité et/ou des donateurs avec des ressources peuvent être d'une importance cruciale pour garantir le succès de la mise en ouvre des deux processus. Au Bangladesh, avec un financement d'USAID, un groupe d'organisations a soutenu le Conseil d'administration pour la planification familiale, comptant Population Council, JSI et Pathfinder International. Après son introduction UNFPA est devenu le partenaire financier principal soutenant le programme du gouvernement de développement à grande échelle. Au Kenya, la fondation William et Flora Hewlett soutient une initiative nationale visant à généraliser les PCU dans les secteurs public et privé. Dans le cadre de cette initiative, Population Council a collaboré avec PSI, Marie Stopes/Kenya, la Pharmaceutical Society of Kenya, plusieurs distributeurs commerciaux de produits de PCU et UNFPA.

6. Le développement à grande échelle devrait être effectué en plusieurs étapes. Après que le projet pilote a fait les preuves que les PCU et leur modèle de délivrance sont acceptables et faisables, il est déconseillé de s'engager tout de suite vers un développement à l'échelle nationale. Un développement à grande échelle progressif est recommandé juste après la phase d'essai ce qui permet de revoir, d'adapter et de modifier si nécessaire les procédures et pratiques développées durant la phase d'essai. Cette première phase d'expansion devrait être suffisamment longue pour apprendre et bien comprendre les problèmes opérationnels et de gestion que posent l'incorporation des PCU dans des systèmes existants et pour identifier les éléments du modèle d'essai qui ne peuvent être réutilisés et devraient être abandonnés (voir le schéma de la démarche par étape page suivante).

\section{Une planification et un suivi rigoureux} de l'approvisionnement et de la logistique sont essentiels. Le soutien logistique est l'un des piliers d'une distribution efficace des PCU, surtout lors de l'introduction et de l'expansion. Les problèmes de logistique les plus couramment rencontrés par des grands systèmes de fourniture englobent entre autres: l'enregistrement du produit et son approvisionnement auprès des fabricants, une mauvaise distribution des PCU ou des stocks d'IEC aux points de fourniture du service, des erreurs de calcul de la demande ou de l'approvisionnement entraînant des ruptures de stocks ou des excédents, et donc des produits périmés. $\mathrm{Au}$ Bangladesh par exemple, de nombreux établissements ayant reçu des matériels d'IEC ne les ont pas distribués aux clients potentiels car leur expérience du système logistique public leur a fait croire qu'ils ne seraient pas réapprovisionnés à temps. Au Kenya, le MS a reçu une importante livraison de PCU avant leur intégration dans le système logistique et dans les registres de planification familiale, par conséquent les produits n'ont pas été distribués systématiquement au niveau des districts et des provinces, mais dans des établissements sans prestataires formés ni de procédures pour le réapprovisionnement (Keesbury, Owino, et Bradford 2007).

8. Planifier et définir un budget pour une aide technique à long terme pour le processus de généralisation, en utilisant de préférence le personnel des phases d'essai ou de celles d'expansion restreinte. L'introduction de n'importe quel nouveau service dans un grand système d'offre requiert certains ajustements et le renforcement des capacités pour garantir la réussite de son intégration. Faire seulement savoir aux responsables de programmes que les PCU sont acceptables et décrire le modèle qui les a introduites et fournies ne va pas leur permettre de les adapter à leurs systèmes. Une assistance technique durable se doit d'être planifiée et budgétisée, avec l'aide de ceux qui connaissent les modèles d'offre des PCU, jusqu'à ce que l'intégration nationale soit réussie. Mettre fin à l'assistance technique avant que la méthode ne soit complètement intégrée peut entraîner une introduction partielle et même une interruption de sa fourniture. Ceci fut le cas au Ghana, où l'association pour la planification familiale (PPAG) a soutenu les efforts du MS pour introduire la CU dans le secteur public à travers une série de projets pilotes. Les bénéfices de ce travail, n'ont cependant pas pu être largement multipliés en raison d'un financement limité de l'aide technique régulière et du plaidoyer de PPAG (Osei 2007).

9. Un suivi rigoureux des services au début de la généralisation est crucial. Tous les éléments d'un projet ou d'une initiative pour introduire les PCU dans le programme national de SR (la 
formation, le conseil, la distribution et la communication) nécessitent un suivi et une documentation systématique pour deux raisons d'égale importance: 1) s'assurer que les activités d'introduction ont été mises en œuvre comme prévu et fonctionnent efficacement; et 2) avoir un dossier sur les facteurs facilitants et les obstacles rencontrés pendant le processus d'introduction afin qu'une action appropriée puisse être entreprise lors du développement à grande échelle et de la généralisation de ces expériences d'introduction. Le suivi de la formation et le développement de systèmes d'aide peuvent être utilisés pour fournir un feedback afin que les formations prévues après la fin de la généralisation puissent se baser sur ces expériences. Le suivi de routine utilisant des données du SIG peut apporter un feedback une fois que les PCU ont été intégrées dans le système de prestation de planification familiale. Ces données peuvent être utilisées pour identifier les établissements de prestation de services ayant besoin de plus de formation, supervision ou de renforcement en général. Mener de brèves évaluations de suivi à mi parcours (par exemple après six mois de prestation de service) peut être utile pour évaluer la qualité des services fournis-par exemple pour savoir si les nouvelles connaissances et compétences ont été retenues.

\section{Le suivi et des évaluations ponctuelles} doivent être maintenus après la généralisation. Trois indicateurs peuvent être utilisés pour déterminer quand les PCU ont été effectivement intégrées dans le système:

$\checkmark$ L'intégration des PCU dans la liste des produits essentiels du système de santé publique afin qu'il y ait toujours une ligne pour ces produits dans le budget pour l'approvisionnement des contraceptifs.

$\checkmark$ L'intégration des PCU dans le système SIG national pour les contraceptifs, qui démontre la volonté de suivre les performances du programme en termes de capacité de fourniture des PCU.

$\checkmark$ Communiquer le nombre et la proportion de prestataires et d'établissements ayant déclaré le stock et la distribution ou la vente de PCU comme l'un de leurs services ou produits habituels.

$\checkmark$ Le suivi devrait continuer après que la fourniture des PCU a été totalement intégrée dans les systèmes de prestation de santé publique, du privé ou des ONG.

Après cela, le suivi ne se focalisera plus sur les informations sur la mise en œuvre des activités d'introduction, mais sur la mesure régulière d'offre des PCU comme l'un des services de PF dans l'ensemble du programme de SR. Des sondages périodiques spécifiques peuvent fournir des données importantes sur les performances des programmes, par exemple, grâce à l'intégration de questions ou de modules sur les PCU dans les sondages de santé reproductive au niveau national et du district. Au Kenya, un tel sondage a été mené après presque une décennie de prestation de services de PCU et a permis aux responsables de programmes de réfléchir sur le succès de leurs efforts et de réorienter leurs stratégies si nécessaire (Keesbury, Owino, et Bradford 2007). De plus, des données sur le rôle de transition des PCU pour augmenter l'utilisation régulière de la PF soutiendront la reconnaissance de l'introduction des PCU et de leur vulgarisation en tant qu'intervention importante de santé de la reproduction. 
Abuabara, K. et al. 2004. "As often as needed: Appropriate use of emergency contraceptive pills," Contraception 69(4):339-342.

Apsel, D. 2007. "Emergency Contraception in Nigeria. A Case Study,” unpublished paper.

Arteaga, Elizabeth and Ivonne La Fuente. 2001. "Las Farmacias como Principales Proveedores de Orientación y Provisión de Métodos Anticonceptivos a Adolescentes" [Pharmacies as the main providers of counseling and contraceptive methods for adolescents], FRONTIERS Report. La Paz, Bolivia and Washington D.C: Population Council.

Bacic, M., A. Wesselius de Casparis, and E. Diczfalusy. 1970. "Failure of large doses of ethinyl estradiol to interfere with early embryonic development in the human species," American Journal of Obstetrics and Gynecology 107(4):531 534.

Beitz, J et al. 2003. "Youth-Friendly Pharmacy Program Implementation Kit: Guidelines and tools for implementing a youth-friendly reproductive health pharmacy program." Seattle: PATH.

Díaz, J. et al. 2007. "Scaling up family planning service innovations in Brazil: The influence of politics and decentralization," in Scaling Up Health Service Delivery: From Pilot Innovations to Policies and Programs, Simmons R., P. Fajans, and L. Ghiron (eds.). Geneva: WHO Press, pp. 135-155.

ECafrique. 2006. Working with the Media to Increase Public Awareness on Emergency Contraception: A Case Study. Nairobi: ECafrique.

Ellertson, Charlotte et al. 2002. "Information campaign and advocacy efforts to promote access to emergency contraception in Mexico," Contraception 66(5):331-337.

Family Health International (FHI). 2007. "How to be reasonably sure a client is not pregnant," a checklist. Research Triangle Park, North Carolina: FHI.

Faundes, A. et al. 2007. "Emergency contraception under attack in Latin America: Response of the medical establishment and civil society," Reproductive Health Matters 15(29):130-138.

Fernandez, C. Araceli and Ricardo Vernon. 2007. "Introduction and expansion of emergency contraception: Lessons from Latin America and the Caribbean for a global guidance tool," unpublished paper.
Fernández, A. et al. 2008. "Introduction and scaleup of emergency contraception products and services: Lessons learned from the field in three continents," unpublished paper.

Givaudan, M., C. Fuertes, and R. Vernon. 1998. "Testing strategies to disseminate knowledge of emergency contraception," INOPAL III Final Report. IMIFAP and Population Council: Mexico and Washington, DC.

Glasier, A. et al. 2004. “Advanced provision of emergency contraception does not reduce abortion rates," Contraception 69(5):361-366.

Goodyear, L. and T. McGinn. 1998. "Emergency contraception among refugees and the displaced," Journal of American Medical Women's Association 53(5 Suppl 2):266-70.

Hatcher, Robert A. et al. 1997. The Essentials of Contraceptive Technology. Baltimore: Johns Hopkins Bloomberg School of Public Health, Population Information Program. International Consortium for Emergency Contraception (ICEC). 2003a. "Regimen Update." $<w w w . c e c i n f o . o r g\rangle$

2003b. "Repeated Use of Emergency Contraception: The Facts." <www.cecinfo.org $>$

ICEC. 2004. "Emergency Contraceptive Pills: Medical and Service Delivery Guidelines." $<w w w$. cecinfo.org>

ICEC. 2005. The Emergency Contraception Newsletter. Winter 2004/2005, Vol. 9, No. 2.

ICEC. 2008. "ECP Status and Activity by Country." $<w w w . c e c i n f o . o r g>$

ICEC. "Adapting Resource Materials for Local Use." <www.cecinfo.org >

ICEC. "Materials for EC Advocacy: Questions and Answers for Decision Makers." $<w w w . c e c i n f o . o r g>$

ICEC. "Materials for EC Clients." <www.cecinfo. org>

ICEC and the International Federation of Gynecology and Obstetrics (FIGO). 2008. "How do levonorgestrel-only emergency contraceptive pills prevent pregnancy?" <www.cecinfo.org $>$

International Family Planning Perspectives. 2004. Editorial, International Family Planning Perspectives 30(4).

International Planned Parenthood Federation/ 
Western Hemisphere Region (IPPF/WHR). 2006. Every Woman's Right: Recommendations for Improving Knowledge and Access to Emergency Contraception. New York: IPPF/WHR.

Keesbury, J., J. Skibiak, and M. Zama. 2006. "Reducing unwanted pregnancy among victims of sexual assault: New windows of opportunity for emergency contraception," unpublished paper.

Keesbury, J., B. Owino, and S. Bradford. 2007. "Emergency contraception, female condoms and IUDs in Kenya's public sector: Findings from a national diagnostic assessment." Nairobi: Population Council.

Keesbury, Jill, Hailegiorgis Aytenfisu, and Spike Bradford. 2007. "Mainstreaming emergency contraception in Ethiopia's public sector." Nairobi: ECAfrique, Population Council, and Ethiopian Society of Obstetricians and Gynecologists.

Keesbury, Jill. 2007. "From pilots to regional programs: Expanding contraceptive choice and improving quality of care in Zambia's Copperbelt: Final project report." Nairobi: Population Council.

Keesbury, J. and M. Zama. 2007. "Emergency contraception for survivors of sexual assault: New windows of opportunity in Zambia," a presentation at the Global Health Conference, Washington, DC, 29 May - 1 June.

Keesbury, J. et al. "Who uses EC in Kenya? An analysis of ECP pharmacy clients," unpublished paper.

Kestler, Edgar and Lilian Ramirez. 2004. Informing the medical community in Guatemala about emergency contraception," FRONTIERS Final Report. Washington, DC: Population Council.

Khan, M.E. and S. Hossain. 2007. "Introducing, promoting, and scaling up emergency contraception in South and Southeast Asia: Regional synthesis paper," unpublished paper.

Khan, M.E. and S.M.I. Hossain. Forthcoming. "Introducing emergency contraceptive pill in the national FP program of Bangladesh: Enhancing utilization of OR results and lessons learned from program implementation," FRONTIERS Final Report. Washington, DC: Population Council.

Khan, M.E. et al. 2008a. "A situation analysis of care and support for rape survivors at first point of contact in India and Bangladesh," FRONTIERS Final Report. Washington, DC: Population Council.

. 2008b. "Introduction of emergency contraceptive pills in the public health system of Pakistan: A south-to-south collaboration," FRONTIERS Final Report. Washington, DC: Population Council.

Khan, M. E and SMI Hossain. 2004. "Introducing emergency contraceptive pills in Bangladesh: A feasibility study," FRONTIERS Final Report. Washington, DC: Population Council.

Kumar et al. 2007. "Provision of emergency contraception services through paraprofessionals in India," FRONTIERS Final Report. Washington, DC: Population Council.

LaNación.cl. 2008. "Al calor de las sábanas municipales [Under city sponsorship]," in LaNacion.cl., 25 May 2008. Santiago. <http://www. lanacion.cl/prontus_noticias_v2/site/edic/2008_ 05_25_1/port-domingo/fk_portada.html>

LaNación.cl. 2007. "Otro debate sobre la píldora del día después. En Ushuaia evitan su distribución [Another debate about the morningafter pill: Avoiding distribution in Ushuaia]," in La Nacion.cl., 3 May 2007. Buenos Aires. <http:// www.lanacion.com.ar/nota.asp?nota_id=905334>

La Nación. 2006. "Polémica discusión: La píldora del día después, gratuita. La extenderán por ley a todo el país [Controversy: The morning-after pill, free: Available country-wide by law]," in La Nación, 16 November 2006. Buenos Aires. $<$ http://www.lanacion.com.ar/nota.asp?nota_ $i d=859258>$

Lara, D. et al. 2007. "Introducing emergency contraception in the Mexican family planning official norm," Gaceta Médica de México 143(6):483-487.

Martin, A. 2004. "Emergency contraception in Latin America and the Caribbean," Revisita PanAmerican de Salad Publica 16(6):424-431.

McGinn, Therese. 2000. "Reproductive health of war-affected populations: What do we know?" International Family Planning Perspectives, 26(4): 174-180.

Ministry of Health and Family Welfare (MOHFW). 2006. Implementation Guide on Reproductive and Child Health II. Adolescent Reproductive Sexual Health Strategy: Handbook for State and District Program Mangers. New Delhi: MOHFW.

Morgan, G. 2007. "A profile of EC Users in Kenya," a presentation delivered to a national EC strategy development workshop, Nairobi, Kenya, 28 August 2007.

Muia, Esther, Fariyal F. Fikree, and Joyce Olenja. 2002. "Enhancing the use of emergency 
contraception in a refugee setting: Findings from a baseline survey in Kakuma refugee camps, Kenya.” Nairobi: Population Council.

Muia, E. et al. 2002. "Evaluation of an emergency contraception introduction project in Kenya," Contraception 66(4):255-260.

Muteshi, J. and J. Keesbury. 2007. "Regional synthesis paper: Emergency contraception: Lessons from Africa," unpublished paper.

Okwemba, A. and J. Anyona. "Having fun with morning-after pills," Daily Nation. 13 May 2004: 25-26.

Osei, I. F. 2007. "Emergency contraception in Ghana: A case study," unpublished paper.

Parker, Chris. 2005. "Adolescents and emergency contraceptive pills in developing countries," FHI Working Paper Series No. WP05-01. Research Triangle Park, North Carolina: FHI.

PATH. 2004. Resources for Emergency Contraceptive Pill Programming: A Toolkit. Seattle: PATH.

Pathfinder. 2007. Emergency Contraceptive Pills, 2nd edition. Watertown, MA: Pathfinder International.

Planned Parenthood Federation of America (PPFA). 1998. Emergency Contraception Handbook. New York: PPFA.

Population Council. 2006. "Facts about emergency contraceptive pills," fact sheet. New York: Population Council.

Population Services International (PSI). 2007. "Products and Services." <www.psi.org/our programs/products/ec.html>. Accessed January 2008.

Raymond, E.G., J. Trussell, and C. Polis. 2007. "Population effect of increased access to emergency contraceptive pills: a systematic review," Obstetrics and Gynecology 109(1):181-88.

Schiavon, R. 2006. “Adolescentes y Anticoncepción de Emergencia” [Adolescents and emergency contraception]. Gaceta Médica de México. 142:6.

Sebastian, Mary P. et al. 2005. "Studying the utilization of emergency contraceptive services through paramedics in India," FRONTIERS Asia and the Near East Region Research Update no. 5. New Delhi: FRONTIERS/Population Council.

Skibiak, John P., Mangala Chambeshi-Moyo, and Yusuf Ahmed. 2001. "Testing alternative channels for providing emergency contraception to young women." Nairobi: Population Council.

Sherestha, Mohindra, M. E. Khan, and Sharif Mohammed Ismail Hossain. 2008. "Strengthening emergency contraception in Nepal," FRONTIERS Final Report. Washington, DC: Population Council.

Taracena, Berta and Carlos Brambila. 2001. "Reproductive health promotion for women victims of violence and rape." Guatemala City: Population Council.

Townsend J. et al. 2007. "EC bridging in Africa: ECAfrique," a presentation at the International Consortium on Emergency Contraception, New York, 4 October.

United Nations Population Fund. (UNFPA). 2001. The Reproductive Health Kit for Emergency Situations, 2nd edition. Geneva: UNFPA.

United Nations Children's Fund. (UNICEF). 2008. "Thuthuzela Care Centres." <www.unicef.org/ southafrica/hiv_aids_998.html>. Accessed March 2008.

UN High Commissioner for Refugees (UNHCR). 2003. "Sexual and Gender-Based Violence against Refugees, Returnees and Internally Displaced Persons: Guidelines for Prevention and Response."<http://www.rhrc.org/pdf/gl_sgbv03_ 00.pdf . Accessed March 2008.

Van Hertzen, H. et al. 2002. "Low dose mifepristone and two regimens of levonorgestrel for emergency contraception: a WHO multicentre randomised trial," Lancet 360(9348):1803-10.

Vernon, Ricardo, Rafaella Schiavon, and Silvia Elena Llaguno. 1997. "Emergency contraception as an element in the care of rape victims," INOPAL Final Report. Washington, DC: Population Council.

Vernon, Ricardo. 1998. "Experiences in the diffusion of emergency contraception in Latin America," in Reproductive Health Operations Research 1995-1998. Washington, DC: Population Council.

Vernon, Ricardo and Maricela Durá. 2004. "Improving the reproductive health of youth in Mexico," FRONTIERS Final Report. Washington, DC: Population Council.

Vernon, Ricardo. 1999. "Mexico: Informing service providers and factory workers about emergency contraception," FRONTIERS Final Report. Washington, DC: Population Council.

World Health Organization (WHO). 1998. New Emergency Health Kit 98. Geneva: WHO. 
WHO and UNHCR. 2001. Clinical Management of Survivors of Rape: A Guide to the Development of Protocols in Refugee and Internally Displaced Persons Situations. WHO and UNHCR.

WHO, UNDP, UNFPA, and World Bank. 2005.

"Levonorgestrel for emergency contraception," fact Sheet. Geneva: WHO, Special Program of Research, Development and Research Training in Human Reproduction.

World Health Organization, Department of Reproductive Health and Research (WHO/RHR) and Johns Hopkins Bloomberg School of Public Health/Center for Communication Programs (CCP) INFO Project. 2007. Family Planning: A Global Handbook for Providers. Baltimore and Geneva: CCP and WHO.

WHO. 2005. "Emergency Contraception," fact sheet no. 244. Geneva: WHO.

WHO. 2003a. Guidelines for medico-legal care for victims of sexual violence. Geneva: WHO.

2003b. "Minimum Initial Service

Package (MISP),” fact sheet. Geneva: WHO.

Youth Net/FHI. 2004. Youth Friendly Services: An Annotated Web- based Guide to Available Resources. Arlington, Virginia: FHI. 


\section{(2) Population Council}

\section{La recherche qui fait une différence}

Le Population Council mène des recherches à travers le monde afin d'améliorer les politiques, les programmes et les produits dans trois domaines: VIH/SIDA; pauvreté, genre et Jeunes; et santé de la reproduction.

\section{FRONTIERS \\ IN REPRODUCTIVE HEALTH}

FRONTIERS est financé par l'Agence des EtatsUnis pour le développement international (USAID)

l'Accord Coopératif Numéro HRN A-00-98-0001200 .

New York

Population Council One Dag Hammarskjold Plaza, New York New York 10017 , USA

Telephone: +1 2123390500

Facsimilie: + 12126052

E-mail:pubinfo@popcouncil.org
Washington

Population Council

4301 Connecticut Avenue, NW

Suite 280, Washington, DC 20008, USA

Telephone: +1 2022379400

Facsimile: $+1202+237+8410$

E-mail: popcouncil@popcouncil.org
India

Population Council

Frontiers in Reproductive Health Program Zone 5A, Ground Floor, India Habitat Centre Lodi Road, New Delhi 110003

Tel: 91-11-2464 2901/2, Fax: 91-11-2464 2903

E-mail: frontiers-india@popcouncil.org

FRONTIERS Website: http://www.popcouncil.org/frontiers/ 